Draft paper. To be published in Atomization and Sprays.

\title{
Subgrid analysis of liquid jet atomization
}

\author{
J. Chesnel ${ }^{\mathrm{a}, *}$, J. Réveillon ${ }^{\mathrm{a}}$, F.X. Demoulina ${ }^{\mathrm{a}}$, T. Ménard $^{\mathrm{a}}$ \\ ${ }^{a}$ CNRS, CORIA UMR 6614, University of Rouen, Technople du Madrillet, BP 12, 76801 \\ Saint-Etienne-du-Rouvray Cedex, France
}

\begin{abstract}
The objective of this paper is to study the feasibility of large eddy simulations of a liquid fuel injection in combustion chambers. To do so, a priori analyses of direct numerical simulations are carried out. A complete liquid jet atomization, from the injector down to the end of the liquid core, is simulated thanks to the coupling of both level-set and VOF formulations. To avoid the apparition of a subgrid term in the right hand side of the continuity equation, the choice was made to consider an incompressible formulation as far as the filtering operator is concerned. The corresponding LES transport equations and various subgrid contributions are thus presented. Results are first dedicated to the estimation of the various orders of magnitude of these subgrid terms. In a second part, classical eddy viscosity scale similarity models are tested against the prevalent ones. It appears that, contrary to a Smagorinsky formulation, the scale similarity assumption provides a better estimation of the subgrid terms. This result is found for all locations that have been considered in the jet: at the injection level or in the atomized area. The major drawback is the presence of a constant that needs to be estimated. Various values are found depending on the filter size.
\end{abstract}

Keywords: atomization, subgrid modeling, Large Eddy Simulation, two-phase flow

\footnotetext{
${ }^{*}$ Corresponding author. E-mail address: jeremy.chesnel@coria.fr
} 
Nomenclature

\section{Roman letters}

n Normal vector to the liquid/gas interface

$\mathbf{u}^{\boldsymbol{\Gamma}} \quad$ Liquid/gas interface velocity vector

u Flow velocity vector

$P \quad$ Local Pressure

$\mathcal{H} \quad$ Heaviside function

Greek letters

$\chi \quad$ Liquid phase function

$\delta_{\Gamma} \quad$ Dirac function centred on liquid/gas interface

$\kappa \quad$ Liquid/gas interface curvature

$\mu \quad$ Local dynamic viscosity

$\Phi^{l s} \quad$ Level-set function

$\Phi^{\text {vof }}$ Volume Of Fluid (VOF)

$\rho \quad$ Local density

$\sigma \quad$ Surface tension coefficient

\section{Operators}

* $\quad$ Spatial filtering operation 


\section{Introduction}

Injection of liquid fuel in combustion chambers is the beginning of many physical interactions involving complex phenomena, starting from the atomization process down to the combustion and exhaust of burnt gases. Combustion regimes and flame structures depend directly on the mixture fraction issued from the evaporation of the liquid phase. Therefore, the atomization process is a key phenomenon controlling the spray dispersion, evaporation and combustion in the chamber.

Development of economically efficient engines, while achieving low noise levels and low pollutant emissions, is a challenge that needs modeling to be fulfilled. Experiments and numerical simulations to study combustion in various application areas have been carried out for several decades [Law, 2006]. Among them, modeling of combustion devices has been at the center of numerous developments for forty years owing to the importance of transportation vehicles [Baumgarten, 2006]. More recently, the strong impact of the presence of a liquid phase prior to combustion has been realized. Since the review paper of [Sirignano, 1983], modeling of evaporating sprays has made several advancements and several issues have been addressed, although some stumbling blocks remain. By going 'upstream' in the combustion chamber, the first major physical phenomenon to be considered, analyzed and modeled is the atomization process. However, because it involves a liquid/gas interface, major difficulties appear from both experimental and numerical points of view and the atomization process remains an open field to explore.

As far as large Weber and Reynolds numbers are concerned, as in many practical situations, there is a lack of knowledge concerning the topology of a liquid jet and its interactions with the surrounding turbulent flow leading to the spray formation. One possible way to analyze the evolution of the liquid phase at the outlet of an injector 
is to use direct numerical simulations (DNS), adapted to the presence of an interface. When monophasic flows are considered, DNS solves the classical Navier-Stokes equations, which consider the fluid as a continuum. Using a fine computational mesh allows the macroscopic structures to be captured since all the considered distances are considerably larger than molecular length and time scales. DNS was first introduced 35 years ago by [Orszag and Patterson, 1972] followed by [Rogallo, 1981; Lee et al., 1991] for the simulation of inert gaseous flows. It resolves all the characteristic scales of a turbulent flow from the Kolmogorov 'dissipative' length scale up to the integral 'energy containing' length scale. However, the apparition of an interface and a strong variation of density jeopardizes the possibility of achieving a complete DNS. This is especially true if fundamental physical phenomena, like evaporation or heat transfers, are present at the interface level. Then, the computational cost of the DNS of the whole flow, including both phases, would be skyrocketing unless some major assumptions were made.

A first possibility is to adopt an interface tracking approach like the 'volume of fluid' (VOF) method developed by [Hirt and Nichols, 1981]. This approach is based on the reconstruction of the gas/liquid interface from the time and space evolution of the local volume fraction of liquid. This mass conservative procedure is complex as far as the interface reconstruction is concerned. Another possibility is to use the level-set (LS) procedure of [Osher and Sethian, 1988]. The procedure follows the motion of an iso-surface of a specific scalar function that maintains algebraic distances. Generally, an incompressible formulation is used but then, evaporation, heat transfer or even combustion phenomena are difficult to account for. Nevertheless, these methods are very promising as demonstrated recently by [Berlemont and Tanguy, 2005] who simulated for the first time the complete atomization of a liquid jet.

DNS allows the surface tension forces of the liquid to be characterized, which 
plays a major role in the atomization processes. These surface tension forces are obtained from the estimation of the curvature of the liquid/gas interface, correctly estimated by DNS, which captures the smallest scales of the flow. However, this implies simulations with a limited domain size and low turbulent Reynolds numbers.

Furthermore, Reynolds Averaged Navier-Stokes (RANS) computations of atomization can be carried out to determine the global impact of the sheared flow. RANS methods are quick and necessary if we set out to model the whole combustion chamber. However, numerous empirical laws are used to predict the spray granulometry, the length of the liquid core and the spray velocity dispersion. This may lead to strong errors as far as the atomization process is concerned, and it will affect all the considered physical phenomena in the chamber. Progress has been made recently by linking the primary breakup Eulerian modeling to a Lagrangian description of the dispersed phase [Demoulin et al., 2007; Lebas et al., 2009; Vallet et al., 2001]. Yet, even if correct results are obtained in given cases, accounting for unstationnary effects and large-scale instabilities remains a challenge.

To overcome these difficulties, the current trend in turbulence modeling is the development of large eddy simulations (LES). LES is a promising technique between industrial RANS solvers and academic DNS solvers. It solves the largest scales of the flow while the impact of the smallest scales (or the subgrid scales) is modeled. Since the development of subgrid dynamic models [Germano et al., 1991; Lilly, 1992], LES of one-phase or two-phase dispersed flows is now widely used in the CFD community. LES proved to be very efficient although it is more expensive than RANS computations. The idea of modeling turbulent flows and interfaces based on a LES procedure is very recent and classic interface tracking methods have been used in LES solvers. With the exception of [Yue et al., 2005], who used the Level-Set procedure, most of the recent work uses the VOF method associated with a LES formulation to simulate 
separated flows as waves [Lubin et al., 2006; Hieu et al., 2004; Christensen, 2006] and bubbles [Deen et al., 2001; Liovic and Lakehal, 2007]. Although, in 2003, [De Villier et al., 2004] have performed one of the first LES of primary diesel spray atomization, the subject is rather unexplored. Moreover, major parts of LES studies that have been carried out consider standard subgrid scale (SGS) models for the momentum balance equation but not for the interface equation. Thus, the impact of the subgrid flow properties on the droplet formation process is neglected and only the large-scale instabilities participate in the spray formation. However, it should be noted that [Chesnel et al., 2010] have proposed a first attempt at complete LES of atomization, which takes into account the interface subgrid term.

Recently, [Labourasse et al., 2007] have performed the first analysis of the subgrid term of the phase function transport equation. In their study, the authors conducted a priori tests on DNS results to provide information on the order of magnitude of the subgrid scale terms applied to bubble deformation. Their main conclusion was that the most important subgrid scale term remains the inertia term, which can no longer be modeled by a classical viscosity model close to the interface. In their subsequent work, [Vincent et al., 2008] continued their analysis with a droplet of oil embedded in water. Other a priori tests have been carried out in [Toutant et al., 2008] with a strongly deformable bubble in a spatially decaying turbulence. This last reference states clearly that a major difficulty arises when considering LES of flows containing discontinuities like interfaces. Indeed, as detailed by [Sagaut and Germano, 2005], subgrid models are generally based on the fact that gradients originate only from the large-scale turbulence and not from the presence of an interface. One solution would be, to set apart the interface impact on the turbulence model by using non-centered filters. However, this procedure could be difficult to carry out from a technical point of view. The solution adopted by [Labourasse et al., 2007; Toutant et al., 2008] was 
to introduce the Interfaces and Subgrid Scales (ISS) as an equivalent to a one-phase LES concept. In this framework, they used centered filters even at the interface level. The subgrid terms issued from the discontinuity presence are accounted for in models.

The objective of the presented research is to extend subgrid-scales a priori analyses to complex two-phase flows thanks to the DNS of the full atomization of a liquid jet. In this framework, the subgrid terms are evaluated in a complete atomization process from the liquid injection down to droplets dispersion. Following the brief presentation of the considered equations and the DNS of liquid jet atomization, we test several closures for the so called subgrid terms. Results are provided with an evaluation of the modeling of the momentum and phase function subgrid terms.

\section{Modeling considerations}

Since the first dynamic model developed by Germano [Germano et al., 1991], LES is seen as the most promising tool concerning the modeling of turbulent flows. Much research has been done for both incompressible and compressible flows. However, as detailed in the introduction, LES of turbulent interfacial flows has rarely been addressed. Thus, in the following, two-phase flow equations are presented and a filtering operator is applied so that large eddy simulation formulation can be detailed. Ultimately, mathematical formulations for the subgrid closures are presented.

It should be noted that indicial notations, via Einstein summation convention, have been adopted instead of the more compact vector notations, since a nonhomogeneous sheared flow has been used in the following. For the sake of clarity, it is important to show the various directions of the tensors. 


\subsection{The 1-fluid formulation}

First and foremost, it is necessary to establish the governing equations of the the two-phase flow. To do so, we start from the so called 1-fluid formulation in this study. The main idea is to obtain a single set of governing equations, which allows us to describe the whole simulation domain (liquid/gas in our case).

Considering two non-miscible fluids (indiced $k=l$ and $k=g$ for liquid and gas, respectively), this formalism introduces a phase function, denoted $\chi_{k}$, to discriminate each phase and to allow for the mass and momentum jump conditions across the liquid/gas interface. This variable, also called 'color function', is defined by $\chi_{k}=1$ for phase $k$ and $\chi_{k}=0$ otherwise. In this study, two phases are considered: liquid and gas. Thus, for the sake of simplicity, $\chi$ represents the liquid phase and $(1-\chi)$ the gas phase. Thus, the local quantities are obtained using the phase function:

$$
\begin{aligned}
\rho & =\chi \rho_{l}+(1-\chi) \rho_{g} \\
\mu & =\chi \mu_{l}+(1-\chi) \mu_{g} \\
\mathbf{u} & =\chi \mathbf{u}_{\mathbf{l}}+(1-\chi) \mathbf{u}_{\mathbf{g}} \\
P & =\chi P_{l}+(1-\chi) P_{g}
\end{aligned}
$$

where $\rho, \mu, \mathbf{u}$ and $P$ are the local density, dynamic viscosity, velocity vector and pressure, respectively.

Following the pioneering works of [Delhaye, 1974], [Drew, 1983] or [Kataoka, 1986], an advection equation is used for the phase function $\chi$, in a classical manner:

$$
\frac{\partial \chi}{\partial t}+u_{i}^{\Gamma} \frac{\partial \chi}{\partial x_{i}}=0
$$


There is no mass exchange between the phases at the interface in our configuration. Consequently, the interface velocity, $\mathbf{u}^{\boldsymbol{\Gamma}}$, and the flow velocity, $\mathbf{u}$, are both equivalent.

Thus, considering the Navier-Stokes equations in each phase $k$, jump conditions and relations 1 to 4, we can build the 1-fluid mass and momentum transport equations system. Especially for the considered case of incompressible, isothermal fluids without evaporation phenomenon, this leads to:

- Mass

$$
\frac{\partial u_{i}}{\partial x_{i}}=0
$$

- Momentum

$$
\frac{\partial \rho u_{i}}{\partial t}+\frac{\partial \rho u_{i} u_{j}}{\partial x_{j}}=-\frac{\partial P}{\partial x_{i}}+\frac{\partial D_{i j}}{\partial x_{j}}-\sigma \kappa n_{i} \delta_{\Gamma}
$$

where:

$$
D_{i j}=\mu\left(\frac{\partial u_{i}}{\partial x_{j}}+\frac{\partial u_{j}}{\partial x_{i}}\right)
$$

The last term on the right hand side of equation 7 represents the surface tension force. Since this force only acts at the liquid/gas interface, $\delta_{\Gamma}$ is the Dirac function related to it. Thus, $\sigma$ denotes the surface tension coefficient, $\kappa$ and $\mathbf{n}$ represent the curvature of the interface and the normal vector of the latter.

Finally, the total governing equations set is made up of equations 6 and 7 associated with relation 5 for interface tracking. It should be noted that this physical model is widely used in the DNS of turbulent two phase flows (e.g. [Menard et al., 2007]). Consequently, many LES a priori studies, based on DNS databases, have characterized the subgrid terms behavior, which appears when filtering these last equations. We particularly mention the works of [Labourasse et al., 2007; Vincent et al., 2008; Toutant et al., 2008] or more recently [Larocque et al., 2010]. 


\subsection{Interface tracking}

From the numerical point of view, resolution of equation 5 requires the development of special procedures generally named interface tracking methods.

To track the interface, various numerical methods exist. A brief summary may be found in [Lakehal et al., 2002] and reference therein. In the following, two of the most used approaches are briefly described: the level-set and the volume of fluid procedure. In fact, these two methods have been combined in order to obtain the DNS database of this study.

\subsubsection{Volume of fluid (VOF)}

The VOF method uses a phase function $\Phi^{v o f}(\mathbf{x}, t)$ to characterize the volume occupied by each phase in a cell. It is defined by the following equation:

$$
\Phi^{v o f}(\mathbf{x}, t)=\frac{V_{l}(\mathbf{x}, t)}{V},
$$

where $V$ is the cell volume and $V_{l}(\mathbf{x}, t)=\int_{V} \chi(\mathbf{x}, t) d V$, the volume of liquid in the cell. Therefore, cells with $\Phi^{\text {vof }}$ included between 0 and 1 contain the interface, the pure liquid phase is designed by $\Phi^{v o f}=1$, whereas $\Phi^{v o f}=0$ indicates a pure gas phase. The evolution of the liquid volume fraction $\Phi^{v o f}$ is characterized by an evolution equation similar to equation 5. The interface location is not directly known and a reconstruction algorithm has to be applied to determine the local surface tension force to be added in the momentum evolution equation. Even if the reconstruction procedure induces severe computational costs, the VOF method presents the advantage of being mass conservative.

\subsubsection{Level-set $(L S)$}

The LS method uses a distance function $\Phi^{l s}(\mathbf{x}, t)$, which provides the algebraic shortest distance of any point $\mathbf{x}$ to the interface. The sign of $\Phi^{l s}$ depends on the 
concerned phase ( $\Phi^{l s}>0$ : liquid phase and $\Phi^{l s}<0$ : gas phase). Thus, the interface corresponds to $\Phi^{l s}=0$. The liquid composition field may be retrieved through the relation:

$$
\chi_{l}(\mathbf{x}, t)=\mathcal{H}\left(\Phi^{l s}(\mathbf{x}, t)\right)
$$

where $\mathcal{H}$ is the Heaviside function. As for the VOF procedure, the time and space evolution of the distance function is governed by an equation similar to equation 5 . This method presents the great advantage of tracking the interface evolution and thus, no reconstruction is necessary. However, mass conservation problems appear frequently.

A promising solution is to associate both LS and VOF methods [Menard et al., 2007]. By doing so, the VOF procedure ensures complete mass conservation of the liquid while the LS method provides the interface position and suppresses the costly reconstruction procedure, especially in $3 \mathrm{D}$ space. This is the procedure we adopted for the DNS of a jet atomization presented in this study. Consequently, both usual procedures have been combined.

\subsubsection{Algebraic properties of the interface}

Topological information about the interface is necessary to determine the contribution of the surface tension forces on the momentum evolution of both phases. Whichever phase function is used $\left(\Phi^{v o f}\right.$ or $\left.\Phi^{l s}\right)$, it is denoted $\Phi$ in the following. In fact, for both formulations, similar expressions allow the definition of the vector normal to the interface:

$$
\mathbf{n}=\frac{\nabla \Phi}{|\nabla \Phi|}
$$

and the interface curvature:

$$
\kappa=-\nabla \cdot \mathbf{n}
$$


Then, $\Phi$ may be considered as the 'mollified color function' as defined by [Brackbill et al., 1992] or a 'smooth approximation' as denoted by [Vincent et al., 2008].

In the following, surface tension forces have been modeled with the Continuum Surface Force (CSF) method, proposed by [Brackbill et al., 1992], using the levelset function, which offers better topological results than VOF (e.g. [Chesnel et al., 2007]).

\subsection{LES transport equations}

\subsubsection{Filtering operation}

As pointed out in the introduction, a formal filtering operation should segregate both phases in order to avoid spurious subgrid information due to the presence of the interface among the turbulence models. However, in order to be able to develop and use models in practical configurations with complex interfaces, it seems difficult to apply filters that would have to be adapted along with the flow evolution. Thus, the interfaces and subgrid scales (ISS) concept [Labourasse et al., 2007; Toutant et al., 2008] seems more adapted: a classical filtering of the turbulent flow is carried out and the interface presence is taken into account in the model definition.

Any field $F(\mathbf{x}, t)$ may be spatially filtered thanks to the relation:

$$
\bar{F}(\mathbf{x}, t)=G \star F(\mathbf{x}, t)
$$

which is a convolution operation over the computational domain volume $\mathcal{V}$ :

$$
\bar{F}(\mathbf{x}, t)=\int_{\mathcal{V}} G\left(\bar{\Delta}, \mathbf{x}-\mathbf{x}^{\prime}\right) F\left(\mathbf{x}^{\prime}, t\right) d \mathbf{x}^{\prime}
$$

The filter function $G(\bar{\Delta}, \mathbf{x})$ allows the definition of a cutoff length scale $\bar{\Delta}$. Several shapes are possible. In our study, a classical top-hat filter has been used: $G(\bar{\Delta}, \mathbf{x})=$ $1 / \bar{\Delta}^{3}$ if $\left|x_{i}\right| \leq \bar{\Delta} / 2, \forall i$; otherwise, the filter function is null. 
From a numerical point of view, the filtering operator used in this study is discretized following:

$$
\bar{F}(i, j, k)=\frac{1}{(2 N+1)^{3}} \sum_{n=-N}^{N} \sum_{m=-N}^{N} \sum_{l=-N}^{N} F(i+n, j+m, k+l)
$$

with:

$$
2 N+1=\left(\frac{\bar{\Delta}}{\Delta}\right)
$$

where $\Delta$ is the DNS mesh size. Thus, since $\Delta$ is constant in time and space in the DNS database, the filtering operator (eq. 15) commutes with time and spatial derivatives [Sagaut, 2003].

Notes on Favre filtering.

As usual, when variable density flows are involved, a Favre filtering operation may be defined

$$
\widetilde{F}(\mathbf{x}, t)=\frac{\overline{\rho F}}{\bar{\rho}}
$$

Therefore, even if the density is constant in each phase, variations of density at the interface level are characterized. However, as stated out by [Labourasse et al., 2007; Toutant et al., 2008], when both phases are incompressible, the Favre filtering operator leads to the disappearance of the divergence free expression (eq. 6).

Instead, a subgrid term appears on the right hand side of the continuity equation:

$$
\frac{\partial \tilde{u}_{i}}{\partial x_{i}}=\frac{\rho_{l}-\rho_{g}}{\bar{\rho}}\left(\overline{\delta_{\Gamma} n_{i} u_{i}}-\overline{\delta_{\Gamma} n_{i}} \tilde{u}_{i}\right)
$$


This term is difficult to close and it could have a strong impact on the whole simulation as noted by [Toutant et al., 2008]. Yet, the incompressibility hypothesis remains identical if operator $\overline{(.)}$ is used:

$$
\frac{\partial \bar{u}_{i}}{\partial x_{i}}=0
$$

A simple way could be to neglect the right hand side of equation 18, as proposed by [Liovic and Lakehal, 2007] on effective LES simulations. However, the preceding authors mentioned the fact that this hypothesis should be used with precaution and assume that this simplification can be made thanks to sufficient time and mesh resolution, in order to capture the liquid/gas interface evolution. Moreover, concerning a priori tests from DNS databases, it seems that this term also depends on the flow configuration. For example, concerning the phase separation flow configuration, [Larocque et al., 2010] has recently shown that this term cannot be neglected.

Thus, whenever possible, Favre filtering has not been used in this paper since no simple solution may be suggested to close the right hand side of equation 18 .

\subsubsection{Filtered 1-fluid formulation}

A filtering operator 14 is applied to equation 5 :

$$
\frac{\partial \bar{\chi}}{\partial t}+\overline{u_{i} \frac{\partial \chi}{\partial x_{i}}}=0
$$

that can be rearranged in

$$
\frac{\partial \bar{\chi}}{\partial t}+\frac{\partial \bar{u}_{i} \bar{\chi}}{\partial x_{i}}+\tau_{\chi}=0
$$

to extract the subgrid correlations between the interface and the velocity field 


$$
\tau_{\chi}=\overline{u_{i} \frac{\partial \chi}{\partial x_{i}}}-\bar{u}_{i} \frac{\overline{\partial \chi}}{\partial x_{i}} .
$$

Physical properties of the flow in the vicinity of the interface depend on the composition field $\chi$. The filtered dynamic viscosity and density fields are defined by

$$
\bar{\mu}=\overline{(1-\chi) \mu_{g}+\chi \mu_{l}}
$$

and

$$
\bar{\rho}=\overline{(1-\chi) \rho_{g}+\chi \rho_{l}},
$$

respectively.

To determine the time and space evolution of both phases, Navier-Stokes equations are resolved. Each phase is incompressible and, as detailed previously, the classic divergence free system may be written, once the filtering operator has been applied:

$$
\begin{aligned}
& \frac{\partial \bar{u}_{i}}{\partial x_{i}}=0 \\
& \frac{\partial \bar{\rho} \bar{u}_{i}+\tau_{\rho u_{i}}}{\partial t}+\frac{\partial \bar{\rho} \bar{u}_{i} \bar{u}_{j}+\tau_{\rho u_{i} u_{j}}}{\partial x_{j}}=-\frac{\partial \bar{P}}{\partial x_{i}}+\frac{\partial \bar{D}_{i j}+\tau_{D_{i j}}}{\partial x_{j}} \\
& -\underbrace{\overline{\sigma \kappa n_{i} \delta_{\Gamma}}}_{\tau_{\sigma_{i}}}
\end{aligned}
$$

with $\bar{D}_{i j}=\bar{\mu}\left(\frac{\partial \bar{u}_{i}}{\partial x_{j}}+\frac{\partial \bar{u}_{j}}{\partial x_{i}}\right)$. The subgrid contributions issued from the filtering of the Navier-Stokes equations are defined by the following expressions: 


$$
\begin{aligned}
\tau_{\rho u_{i}} & =\overline{\rho u_{i}}-\bar{\rho} \bar{u}_{i}, \\
\tau_{\rho u_{i} u_{j}} & =\overline{\rho u_{i} u_{j}}-\bar{\rho} \overline{u_{i}} \overline{u_{j}}, \\
\tau_{D_{i j}} & =\mu\left(\frac{\partial u_{i}}{\partial x_{j}}+\frac{\partial u_{j}}{\partial x_{i}}\right)-\bar{D}_{i j}, \\
\tau_{\sigma_{i}} & =\overline{\sigma \kappa n_{i} \delta_{\Gamma}} .
\end{aligned}
$$

Using the name convention established by [Toutant et al., 2008], we shall use the following denomination for the subgrid terms:

$$
\begin{array}{ll}
\tau_{\chi} & : \text { " } \text { Interfacial term" } \\
\tau_{\rho u_{i}} & : \text { "Temporal term" } \\
\tau_{\rho u_{i} u_{j}} & : \text { "Convective term " } \\
\tau_{D_{i j}} & : \text { : } " \text { Diffusive term" } \\
\tau_{\sigma_{i}} & : \text { "Surface tension term" }
\end{array}
$$

All of these subgrid terms are due to two-phase flow consideration, except the convective term, $\tau_{\rho u_{i} u_{j}}$, which is present in standard single-phase LES equations. $\tau_{\rho u_{i}}$ and $\tau_{D_{i j}}$ are due to the discontinuities of $\rho$ and $\mu$ across the interface, respectively. The two last terms, $\tau_{\chi}$ and $\tau_{\sigma_{i}}$ originate from subgrid interfacial deformations. This can be better understood if we recall that $\partial \chi_{k} / \partial x_{i}=-n_{i}^{k} \delta_{\Gamma}$ in the 1-fluid formulation.

On the one hand, previous a priori evaluations of these various terms suggest that the convective term, $\tau_{\rho u_{i} u_{j}}$, must systematically be taken into account through a model. On the other hand, the behavior of the specific two-phase flow subgrid terms strongly depends on flow configuration (see [Labourasse et al., 2007; Vincent et al., 2008; Toutant et al., 2008; Larocque et al., 2010]). 


\section{DNS of the atomization of a liquid jet}

Using the numerical procedure presented in [Tanguy et al., 2007], the DNS of the complete atomization of a liquid jet has been carried out. This geometry presents several advantages. First, it is very close to experimental and industrial considerations where sheared flows are common. Indeed, jet injection is one of the main ways to atomize a liquid into a spray of droplets and, as stated in the introduction, there is a lack of information concerning the modeling of the area close to the injector. Therefore, some useful information concerning atomization modeling could be derived from this configuration. Analyses are carried out starting from the undisturbed liquid sheet at the outlet of the injector down to a dense spray beyond the disappearance of the liquid core. A wide range of various interface topologies is encountered. Thanks to the DNS, it is thus possible to study them and to evaluate the capability of the models to capture their properties.

\subsection{Configuration}

The basis of Level Set methods has been proposed by [Osher and Sethian, 1988]. The interface is described with the zero level surface of a continuous function defined by the signed distance to the interface. To ensure that the function remains the signed distance to the interface, a re-distancing algorithm is applied. However, it is well known that its numerical computation can generate mass loss in under-resolved regions. To describe the interface discontinuities, a Ghost Fluid Method (GFM), as derived by [Fedkiw et al., 1999], has been carried out to capture jump conditions at the interface. The GFM not only avoids the introduction of a fictitious interface thickness, but it is also suitable to provide a more accurate discretization of discontinuous terms, reducing spurious currents and improving the resolution on the pressure jump condition ([Kang et al., 2000; Berlemont and Tanguy, 2005]). In the 
primary break-up of a jet, numerous topological changes occur: interface pinching or merging, droplet coalescence or secondary break-up. The numerical method should describe the interface motion precisely and handle jump conditions at the interface without artificial smoothing. Moreover, the method should be mass conservative. In the computations carried out in this study, interface tracking is performed by a Level Set method. GFM is used to accurately capture sharp discontinuities, and a coupling between Level Set and VOF methods is made to ensure mass conservation ([Sussman and Puckett, 2000; van der Pijl et al., 2005]). A projection method is used to solve incompressible Navier-Stokes equations, which are coupled to equation 5 for the level set function. A detailed description of the numerical methods may be found in [Menard et al., 2007].

The main properties of the configuration are the following: the size of the domain is $(2.4 \mathrm{~mm}, 0,3 \mathrm{~mm}, 0,3 \mathrm{~mm})$, where the first dimension is the streamwise direction and the other two, the spanwise directions (fig. 1). At the injection level, the jet diameter $D_{0}$ is equal to $0.1 \mathrm{~mm}$, while the liquid jet Reynolds number is equal to $R_{e}=4659$. Turbulence fluctuations are prescribed by using the method developed by [Klein et al., 2003] with a characteristic correlation scale equal to a tenth of the diameter of the injector. A summary of the physical parameters, for this configuration, can be found in table 1 .

Various stages of the injection process have been plotted in figure 2. Liquid surface instabilities close to the injector are visible. Their deformation leads to the formation of ligaments and droplets of various sizes. At the end of the domain, the liquid core has almost disappeared and a dense spray of droplets leaves the computational domain.

Concerning the smallest droplets, it is assumed that no breakup occurs below a local gaseous Weber number value of 10 . This critical Weber number, defined as 
$W e_{G c}=\rho_{g} U_{\text {max }}^{2} D_{g c} / \sigma$, leads to a minimum droplet diameter equal to $D_{g c}=2.4 \mu \mathrm{m}$. Thus, a $2048 \times 256 \times 256$ Cartesian grid has been used with regularly spaced nodes $(\Delta=1.17 \mu m)$.

In the following, a priori subgrid analyses are carried out to evaluate the capability of LES formulations to capture the main characteristic of such an atomizing jet. Three analysis areas have been defined: the injection area at a streamwise position starting from $0 D_{0}$ to $4 D_{0}$ (zone 1 ), the central area positioned from $8 D_{0}$ to $12 D_{0}$ (zone 2) and eventually the atomized area positioned from $20 D_{0}$ to $24 D_{0}$ (zone 3 ). These test zones are also visible in figure 1.

\subsection{Phase function filtering}

As defined in equation 14, a 'top-hat' filtering operator is applied to the various fields of the direct numerical simulation. To begin with, the composition field $\chi$, which characterizes the liquid phase (eq. 5), has been filtered. Two filter sizes have been considered: a small filter size (SFS) $\bar{\Delta}_{5}=5 \Delta$ and a large filter size (LFS) $\bar{\Delta}_{13}=13 \Delta$, where $\Delta$ is the uniform discretization step of the DNS Cartesian grid.

When scrutinizing figure 2, several questions arise concerning the capability of LES models to reproduce such a system. Indeed, the objective of atomization modeling is to provide accurate characteristics of the spray downstream the injector; mainly, the droplet size and velocity distributions.

[De Villier et al., 2004; Buonfiglioli and Mendonca, 2005; Bianchi et al., 2007] have conducted the first LES attempts of atomizing a liquid/gaz jet by using classical closure for the convective subgrid term in the momentum equation and the interface tracking method developed for DNS. In the following we will call this procedure 'Interface Coarse DNS' (ICD).

However, actual use of LES for interfacial flows neglects the impact of subgrid 
phenomena on the phase function and, though mass conservation is well respected, the smallest droplets visible in figure 2 cannot be directly characterized.

Figure 3 presents a cut of the atomizing jet along the streamwise direction. It shows the composition field $\chi$ in three cases: DNS, and then SFS and LFS filtering. The top image presents the DNS composition field with the presence of primary liquid instabilities and the formation of droplets and ligaments as described for figure 2. As soon as a filter is applied, the neat border between liquid and gas phases disappears and the smallest liquid structures vanish to be replaced by a smooth phase function field that could be tracked on a mesh with much less grid points. We will focus on the white lines in figure 3, representing the interface position that would be defined by a large-scale computation. The key problem of large eddy simulations of interfacial flows lies in this picture; in fact, the interface is represented only at a large-scale level. Therefore, due to grid implicit filtering, the phase function frontier (white line) is no longer able to capture the complete liquid evolution. This point is clearly exposed in figure 4, which represents several flow density cuts perpendicular to the streamwise direction. The first line of figures $4-(a-b-c)$ represents the flow density $\rho$, obtained from the DNS computation for the three representative positions: injection, central and atomized areas. In the framework of this analysis, this field may be considered as an exact representation of the researched solution. The second line of figures 4(d-e-f) denotes the application of equation 23 defining the local density thanks to a smoothed composition field. A lot of information is preserved and the presence of ligaments and droplets at the subgrid level is still visible, even if the subgrid liquid geometry is lost.

The limitation of using a composition field with an ICD formulation in a LES computation is clearly visible in figures $4-(g-h-i)$. In these figures, the density field is reconstructed using the sharp interface function related to larger scale, $\varepsilon$ : 


$$
\bar{\rho}(\varepsilon)=(1-\varepsilon) \rho_{g}+\varepsilon \rho_{l},
$$

For our purpose, $\varepsilon$ is reconstructed thanks to the level 0.5 of $\bar{\chi}$ :

$$
\varepsilon=\mathcal{H}\left(\frac{1}{2}-\bar{\chi}\right)=\left\{\begin{array}{l}
1 \text { if } \bar{\chi}>1 / 2 \\
0 \text { otherwise } .
\end{array}\right.
$$

It should be noted that choosing the $\bar{\chi}=0.5$ level is an arbitrary assumption. In ICD-LES simulations, mass conservation is guaranteed by the resolution of an advection equation for $\varepsilon$ similar to equation 5 related to the interface tracking methods developed for DNS.

In ICD formulation, only information about the main liquid core is known and, because of mass conservation, inclusions smaller than the LES grid could not be created and large liquid volumes are not ruptured. These conclusions emerge when scrutinizing the shown $\bar{\rho}(\varepsilon)$ and ICD results in the field of liquid/gas atomization [De Villier et al., 2004; Buonfiglioli and Mendonca, 2005; Bianchi et al., 2007]. In a complete LES framework, the evolution of the interface cannot be treated without taking into account the presence of the liquid phase at the subgrid level.

Clearly, two solutions can be envisioned. A first possibility, which has been adopted in this study, is to work with a smoothed composition field, fig. 4-(d-e-f). A second solution would be to use a classic composition field defining a large-scale interface, as presented in figures 4-(g-h-i). This composition field could be characterized thanks to a conventional VOF or LS phase function. The subgrid properties of the liquid could be represented thanks to a new Eulerian variable defining the subgrid liquid density. Owing to information provided by DNS, this quantity has been plotted in figures $4-(\mathrm{j}-\mathrm{k}-\mathrm{l})$. However, an evolution equation would be necessary and exchange terms with the large-scale composition field have to be determined. 
Therefore, by associating fields 4-(g-h-i) and fields 4-(j-k-l) complete large-scale level information can be obtained. In this paper, for the sake of simplicity, the use of a smoothed composition field $\bar{\chi}$ has been adopted. But the second solution could be an interesting approach to explore, especially if industrial solvers are concerned.

Three main discussions arise concerning the LES of jet atomization. First of all, we could question whether actual LES models are able to capture the correct time and space evolutions of the filtered phase function field, such as the ones obtained in figures 3 and 4 . The objective of this paper is to provide a preliminary answer to this question thanks to an a priori analysis. However, confirmation will be necessary based on effective a posteriori computations. This will be the purpose of future research. A second fundamental question appears when considering the liquid phase. Indeed, one of the main objectives of atomization modeling is to provide information about droplet size and velocity distributions. Therefore, it is necessary to reconstruct this information from the LES field information. A possible method is to use a 'defiltering' (also termed deconvolution) procedure [Sagaut, 2003], or to adopt a statistical description of the properties of the subgrid liquid phase using data provided by the filtered phase function. A last stumbling block concerns the destabilization of the jet. In fact, surface instabilities appearing after injection drive the destabilization of the jet. This leads to various atomization regimes depending on the injection velocity, along with the liquid viscosity and density as well as the surface tension, through the Reynolds and the Weber numbers [Reitz, 1978; Faeth, 1991]. As a result, either LES should be refined to a DNS near the injector to capture the liquid destabilization correctly, or specific subgrid models have to be developed to ensure a correct jet destabilization. 


\subsection{A priori analysis of the subgrid terms}

The objective of the following section is to evaluate the order of magnitude of the subgrid terms present in the LES momentum and phase function equations. A similar analysis has been conducted by [Labourasse et al., 2007] in the framework of a bubble interacting with counter-rotating vortices and by [Vincent et al., 2008] or [Larocque et al., 2010] for a water/oil inversion problem. An extension of their a priori analysis is thus applied to the fully atomized jet in this study.

Thanks to the cylindrical symmetry, it is possible to carry out a radius dependant analysis where data are averaged along the streamwise direction over a short distance $l_{x}$ considered as homogeneous. The following averaging process has thus been employed to analyze any field $A(x, y, z)$ defined on the computational grid:

$$
\langle A\rangle\left(r_{0}, x_{0}\right)=\frac{\int_{r_{0}}^{r_{0}+l_{r}} \int_{x_{0}-l_{x} / 2}^{x_{0}+l_{x} / 2} A r d r d x}{\int_{r_{0}}^{r_{0}+l_{r}} \int_{x_{0}-l_{x} / 2}^{x_{0}+l_{x} / 2} r d r d x},
$$

where $l_{x}$ is the streamwise analysis length fixed to $4 D_{0}, l_{r}$ corresponds to the grid step along the radial direction and the radius $r=\sqrt{y^{2}+z^{2}}$. A similar averaging operator has been used to define interfacial statistics:

$$
\langle A\rangle^{\Gamma}\left(r_{0}, x_{0}\right)=\frac{\int_{r_{0}}^{r_{0}+l_{r}} \int_{x_{0}-l_{x} / 2}^{x_{0}+l_{x} / 2} A \delta_{\Gamma} r d r d z}{\int_{r}^{r+l_{r}} \int_{x_{0}-x_{z} / 2}^{x_{0}+l_{x} / 2} \delta_{\Gamma} r d r d z},
$$

To begin with, the mean turbulent kinetic energy $\langle k\rangle$ and the corresponding dissipation rate $\langle\varepsilon\rangle$ have been plotted in figure 5 . These data have been extracted from the DNS fields; no filter has been applied yet. Close to the injector, turbulent kinetic energy (fig. 5-a ) presents a usual profile with a peak appearing in the area where the shear is at maximum. In our configuration, this corresponds to the interface position. Then, while the jet is destabilized, turbulent structures develop and the global level 
of kinetic energy decreases. The central and atomized area are thus the location of turbulent energy production. In this part of the domain, it is possible to observe a homogenization of the turbulence along the radial direction. Similarly to the kinetic energy, the dissipation rate (fig. 5-b) presents a single peak in the injection area. However, the dissipation level decreases along the streamwise direction while its profile widens because of the jet destabilization and atomization. The final section of our computational box corresponds to the end of the main liquid core atomization. The domain is therefore too short to observe the decay of energy that will appear. Logically, the corresponding level of dissipation remains of low energy production in this area.

More details may be seen in figure 6, which represents the normalized turbulent kinetic energy spectrum close to the injector, in the central area and in the atomized area. Close to the injector, the turbulence is not yet fully developed but, very quickly, an inertial range appears. Its slope respects the usual $k^{-5 / 3}$ of the Kolmogorov theory.

When the filtering process is applied, it is possible to set apart the subgrid kinetic energy from the resolved one, noted $\bar{k}$. The proportion of resolved kinetic energy compared to the total energy extracted from the DNS is plotted in figure 7 for the two filter sizes $\operatorname{SFS}\left(\bar{\Delta}_{5}=5 \Delta\right)$ and $\operatorname{LFS}\left(\bar{\Delta}_{13}=13 \Delta\right)$, previously selected. In figure 6 , the SFS filter position corresponds to $k / k_{\max }=0.2$ and the LFS filter corresponds to $k / k_{\max }=0.076$.

The left plot in figure 7-(a) shows that the smallest proportion of resolved energy may be observed close to the injector in the liquid phase $\left(r / r_{0}<1\right)$. The gas phase is still quiescent and a null energy may be measured (fig. 5-(a)). In the liquid phase, even if the global energy remains weak compared to the peak observed at the interface, turbulent motion is important because it participates in the surface destabilization and atomization. If the SFS filter is applied, this ratio of large-scale 
energy starts from $80 \%$ in the liquid close to the injector and goes up to $90 \%$ far from the jet. This confirms that SFS filtering is useful for a progressive analysis of subgrid filter scales but useless for an effective LES computation. Most of the energy is resolved and the computational cost would be almost similar to a DNS computation. However, as appears in figure 7-(a) if LFS is applied, only $20 \%$ of the energy is resolved at the center of the liquid jet outside the injector because the largest turbulent liquid structures in the injector remain small compared to the considered filter size. After injection, the jet expands and the size of the turbulent structures increases in the liquid area $\left(r / r_{0}<1\right)$. Thus, resolved energy first increases from $20 \%$ to $50 \%$ in the LFS profile in figure 7 -(a). Then, because of the presence of the interface $\left(r / r_{0}=1\right)$, a sudden drop down to $20 \%$ of resolved energy may be observed again. After this drop, resolved energy rises abruptly up to almost $100 \%$, which corresponds to the quiescent atmosphere area. Far from the jet, it is possible to observe (fig. 7-(b) ) that the flow becomes homogeneous along the radial direction and the resolved energy reaches a constant $60 \%$ proportion. A first remark concerns the constant filter size that has been considered. Subgrid models have to reproduce a wide range of unresolved energy: between $80 \%$ in the liquid phase outside the injector down to $40 \%$ after atomization. This wide range implies a double constraint on the filter size: It must be selected within the inertial range and it must be able to represent subgrid energy in the whole calculation domain. Note that a similar discrepancy between injection and far field exist also in monophasic jets and it has been proven that LES is able to capture them [Wang et al., 2008]. However, we have to keep in mind that, in our case, initial liquid perturbations contribute to the jet atomization and the final size distribution of the droplets. The resolved energy drop in the vicinity of the interface shows that small-scale structures are prevalent in this area. 
The complete subgrid budget of the momentum equation is considered in figure 8. In this figure, the four temporal $\left\langle\partial \tau_{\rho u_{i}} / \partial t\right\rangle$, convective $\left\langle\partial \tau_{\rho u_{i} u_{j}} / \partial x_{j}\right\rangle$, diffusive $\left\langle\partial \tau_{D_{i j}} / \partial x_{j}\right\rangle$ and interfacial terms $\left\langle\tau_{\sigma_{i}}\right\rangle^{\Gamma}$ defined in equation 25 have been plotted for positions close to the injector (zone 1) and far from it (zone 3). Note that we chose to work directly with the subgrid term $\tau_{\sigma_{i}}=\sigma \overline{\kappa n_{i} \delta_{\Gamma}}$, since a resolved interface is not clearly defined.

Initially, as for the turbulent kinetic energy evolution, the flow is strongly nonhomogeneous in the radial direction close to the injection. However, as soon as atomization occurs, profiles along the streamwise direction become uniform (fig. 8(b) and (d)). Wherever we are along the streamwise direction, the hierarchy between the various subgrid terms remains similar. At first, temporal and convective terms are prevalent. The diffusion term is much smaller, with a difference of several orders of magnitude. It appears that the interfacial term is very small compared to the others, even the diffusion term. These remarkable properties have been observed previously by [Labourasse et al., 2007] in the framework of the deformation of a bubble. In their study, [Labourasse et al., 2007] suggested that the exceptionally high level of the temporal term may be due to the low order of the time filtering derivative. They were in fact using a first order Euler scheme. To check this hypothesis, it has been decided in this study to use a fourth order temporal scheme. However, it appears that similar conclusions may be drawn and the high magnitude of the temporal term is not the consequence of the numerical scheme. In the work of [Labourasse et al., 2007] the increase of the order of magnitude of the temporal scheme is directly correlated to the deformation of the bubble: the more it is distorted, the higher the temporal term. In our configuration, the liquid interface is much more deformed than the considered bubble and thus, the temporal term remains strong wherever we are in the streamwise direction. In fact, the more the interface is deformed, the 
more it is possible to observe locally a brutal evolution of the flow density at the subgrid level. Additionally, because of the high ratio of density between the liquid and the gas phase, even a slight modification of the interface position may lead to a high value of the temporal term, which represents the subgrid evolution of the correlations between velocity and density.

Examples of convective terms close to the injector have been plotted in figure 9 . Figures 9-(a-b) represent a streamwise-spanwise term $\left(\tau_{u_{1} u_{2}}\right)$ and figures 9-(c-d) a spanwise-spanwise term $\left(\tau_{u_{2} u_{2}}\right)$. Subgrid convective terms appear mainly around the smoothed interface (represented by the thick isoline), corresponding also to the area where the gradients reach their highest level. Instantaneous fields of the tensor have been plotted to demonstrate that the interface presence has a remarkable effect on the subgrid tensor. This is due to the appearance of small structures close to the interface.

Resolved $\langle\overline{\mathbf{u}} \cdot \nabla \bar{\chi}\rangle$ and subgrid convective terms $\left\langle\tau_{\chi}\right\rangle$, of equation 20, have been plotted in figure 10 for two positions: close to the injector and in the atomized area. It appears that the interface convection is mainly controlled by large-scale convection phenomena. In fact, the resolved part is often prevalent. However, the subgrid contributes up to $10 \%$ of the resolved contribution, and is thus not negligible for all the tested zones and filter sizes.

Several authors in previous works, [De Villier et al., 2004; Buonfiglioli and Mendonca, 2005; Bianchi et al., 2007], have chosen to neglect the subgrid scales impact on the interface propagation in liquid/gas atomization flow simulations. Consequently, these authors highlight a strong impact of the mesh resolution on the jet destabilization process as well as on the obtained droplet size distribution.

Close to the injector, the convective subgrid scale contributions could initiate the initial interface destabilization process as noted by [Herrmann and Gorokhovski, 
2008] even on large Weber number flows. As shown in figure 10-(a), for example, there is a sudden growth of the subgrid term at the interface. It appears that the interface subgrid term $\tau_{\chi}$ may necessitate a model, at least in the atomization area.

Starting from the conclusions drawn in the previous section, we propose to test some usual closure for the predominant subgrid terms in the following section.

\section{Subgrid modeling}

In order to carry out large eddy simulations of two-phase flows, subgrid terms, defined in the preceding section, have to be modeled. The aim of this section is to conduct some tests to know which closure should be used for the large eddy simulation of two-phase flows.

We want to evaluate the subgrid term contributions as they appear in the evolution equations of the phase function (eq. 20) and the momentum (eq. 25). Following the estimation of the subgrid terms importance in the previous section, we then focused on the closure of the prevalent terms of the momentum and phase function equations:

- Temporal term contribution, $C_{i}$ :

$$
C_{i}=\frac{\partial\left(\overline{\rho u_{i} u_{j}}-\bar{\rho} \bar{u}_{i} \bar{u}_{j}\right)}{\partial x_{j}}
$$

- Convective term contribution, $T_{i}$ :

$$
T_{i}=\frac{\partial\left(\overline{\rho u_{i}}-\bar{\rho} \bar{u}_{i}\right)}{\partial t} .
$$

- Convective term of the phase function equation contribution, $\Pi$ :

$$
\Pi=\overline{u_{i} \frac{\partial \chi}{\partial x_{i}}}-\bar{u}_{i} \overline{\frac{\partial \chi}{\partial x_{i}}}
$$


It should be noted, that results are presented in dimensionless form, using the factors presented below:

$$
C_{i}^{*}=\gamma C_{i} \quad ; \quad T_{i}^{*}=\gamma T_{i} \quad ; \quad \Pi^{*}=\xi \Pi,
$$

where $\gamma=D_{0} / \rho_{l} U_{d}^{2}, \xi=D_{0} / U_{d}$ and $\xi=D_{0} / U_{d}$. For the sake of clarity, the dimensionless notations $\left(^{*}\right)$ are dropped. Thus, results and graphics presented in the following are systematically non dimensional.

At first, the exact contribution is plotted against the modeled one in dispersion diagrams for each tested model. From a practical point of view it is not possible to plot all the points in each zone. Two data vectors were then used $\mathbf{D}^{\mathbf{e}}$ (exact) and $\mathbf{D}^{\mathbf{m}}$ (model), containing $n$ statistical sample points, determined in a random manner. The $k^{t h}$ component of these vectors contains exact and modeled subgrid contributions taken at the same space point $\mathbf{X}$, and time $t$..

\subsection{Preliminary results}

In the framework of one-phase turbulent flows, classic eddy viscosity models are generally suggested to close the convective term. However, it appears that they are ill-adapted to model subgrid scales of two-phase flows. In their pioneering work, [Labourasse et al., 2007] reach the same conclusions after having tested the Smagorinsky model[Smagorinsky, 1963], the Wale model [Nicoud and Ducros, 1999] and mixed scale models [Sagaut et al., 1999]. In addition, a dynamic Smagorinsky model was tested [Germano et al., 1991] in the framework of our jet atomization configuration and results were far from being satisfactory.

Since the Smagorinsky model has been initially developed for the convective term, we will test it first. It is expressed as follows: 


$$
C_{i}^{m} \simeq-2 \frac{\partial C_{s} \bar{\rho} \bar{\Delta}^{2}|\bar{S}| \bar{S}_{i j}}{\partial x_{j}},
$$

where $C_{s}$ is the Smagorinsky coefficient, $S_{i j}=0.5\left(\partial \bar{u}_{i} / \partial x_{j}+\partial \bar{u}_{j} / \partial x_{i}\right)$ is the resolved strain rate tensor and $|\bar{S}|=\sqrt{2 S_{i j} S_{i j}}$. Classically the Smagorinsky coefficient can be directly prescribed at a constant value $\left(C_{s} \simeq 0.01\right)$, or can be determined by dynamic procedure ([Germano, 1992; Lilly, 1992]). Both methods have been tested in this study. As presented with the LES transport equations $(24 ; 25), \rho$ is not a transported quantity. It is estimated (eq. 23) from the phase function evolution. In figure 11, the modeled contribution of the dynamic procedure has been plotted against the exact contribution for the SFS filter size. Both injections (fig. 11-(a-b)) and atomized (fig. 11-(c-d)) areas have been observed to evaluate the capability of the model for various liquid densities, as for spanwise (fig. 11-a-c) and streamwise (fig. 11-b-d) directions.

From a qualitative point of view, these scatter plots show the difficulty in finding a clear correlation between the exact and the modeled contributions, whatever the observed direction. In all tested cases of this model, the scatterplot is strongly dispersed. This dispersion is worse when the filter size is large (not shown).

It appears that mixed models [Boivin et al., 2000; Toutant et al., 2008], which include a scale similarity assumption, have a much better behavior as far as interfacial two-phase flows are concerned. As mentioned and shown before, it appears that eddy viscosity models are not adapted to model turbulent interfacial flows. These models have been developed in the framework of one-phase turbulent flows without any local discontinuities. The presence of an interface jump induces subgrid fluctuations, which are not directly correlated to the turbulent motion, and leads to a failure of the model. However, scale similarity models do not consider any local properties 
of the turbulence. Indeed, they are based on the projection of resolved fluctuations onto an unresolved grid.

Consequently, scale similarity assumptions are tested in the subsequent research. The closures of the prevalent subgrid terms are then written:

- Convective term contribution closure:

$$
C_{i}^{m}=C_{c}\left(\frac{\partial \bar{\rho}{\widehat{\overline{u_{i}}}}_{\bar{u}}-\widehat{\bar{\rho}} \widehat{\bar{u}}_{i} \widehat{\bar{u}}_{j}}{\partial x_{j}}\right)
$$

- Temporal term contribution closure:

$$
T_{i}^{m}=C_{t}\left(\frac{\partial \widehat{\bar{\rho} \bar{u}_{i}}-\widehat{\bar{\rho}} \widehat{\bar{u}}_{i}}{\partial t}\right) .
$$

- Convective term of the phase function equation contribution:

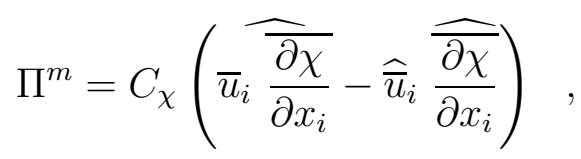

where $\widehat{(\bullet)}$ is a test filtering operator, such as $\widehat{\Delta} / \bar{\Delta}=2$.

One of the main drawbacks of this formulation is the apparition of a projection coefficient between the resolved and unresolved perturbations. Contrary to usual eddy viscosity dynamic models, there is no possible dynamic estimation of $C$. Thanks to the DNS field, an estimation of this parameter is proposed in the following. Its dependence on the filter size but also on the interface properties (continuous or atomized) is scrutinized.

All results of the scale similarity closures have been plotted in figures 12, 13 and 14 for the convective and temporal terms of the momentum equation and the convective 
term present in the evolution equation of the interface, respectively. Both injection (a-b) and atomized (c-d) areas have been observed to evaluate the capability of the model for any liquid configurations. It appears that, for all the subgrid terms, the scale similarity assumptions offer a good agreement. All scatter plots between the effective subgrid terms and their closures present a good correlation, contrary to the Smagorinsky model: figure 12 has to be compared with figure 11. Similar conclusions arise for the LFS filter size (not shown).

As aforementioned, the main drawback of the scale similarity model concerns the estimation of the constant. It is, indeed, not possible to use a dynamical estimation of this constant. Thanks to the DNS data, the values of the various constants of the model as a function of the filter size were estimated. As an example, the results for the convective term coefficient, $C_{c}$ are shown in figure 15 . The ratio of the filter size to the DNS grid has been tested up to 20, although it is far from any physical value we would effectively use. It is possible to see that, in the range of interest (between 5 and $13 \bar{\Delta}$ ), the relation between $C_{c}$ and $\bar{\Delta}$ is quasi-linear. Another important point to note is that the values of the coefficients strongly depend on the position in the jet.

While the two coefficients in the spanwise directions have very similar behaviors in zone 1 to 3 , the streamwise direction, which constitutes a particular direction in this flow, is singular, especially in zone 1.

Therefore, the scale similarity model seems to be the optimal choice to carry out LES of two-phase flows with the presence of an interface. Some values of the various subgrid constants have been proposed, but depending on the configuration and the atomization level, they may need some adjustment. An ideal possibility would be to develop a model using a dynamic procedure to determine its coefficients. However, the tests that have been performed up to now with a dynamic model are 
not satisfactory. In fact, the presence of the interface generates spurious subgrid information.

Moreover, it can be noted that the simple observation of the preceding scatter plots allows, from a qualitative point of view, the different closures to be discriminated but does not state their quality. Indeed, even if the scale similarity assumption shows better behavior than the eddy viscosity closure, it can be observed in figures 12, 13 and 14, that the results do not perfectly fit the exact contributions obtained directly from the DNS. This is illustrated in figure 16, which shows an instantaneous comparison between the exact streamwise contribution of the convective subgrid term (a) and its scale similarity counterpart (b) in spanwise-spanwise cut in zone 1. From a qualitative point of view, this closure shows good behavior compared to the exact contribution. Still, due to the model error, point to point discrepancy can be observed by comparing exact and modeled contributions along the $y$ profile in figure 16-(c).

To advance further, a characterization of the statistical dispersion of the error committed by the tested models is proposed in the following sub-section.

\subsection{Statistical dispersion of the models}

To characterize the statistical dispersion of the error committed by the various models we propose to use an operator, denoted $\sigma$, on the results. An optimistic estimation would be to suppose a certain compensation of the error. Here we opt for the pessimistic evaluation, assuming an accumulation of the error. Thus, for our purposes, we use the quadratic mean which permits to sum the error on the statistical sample. Denoting $\mathbf{D}^{\mathbf{A}}$, vector of sample data, and $n$, the number of samples, $\sigma$ is written as: 


$$
\sigma(A)=\sqrt{\frac{1}{n} \sum_{k=1}^{n}\left(D_{k}^{A}\right)^{2}} .
$$

Using this last operator, we can define a statistical error, $\varepsilon$, in order to characterize the dispersion between the exact subgrid terms and the modeled ones. For instance we can use:

$$
\varepsilon=\sigma\left(\Lambda_{i}^{\prime}-\Lambda_{i}^{m \prime}\right)
$$

where $\Lambda_{i}^{\prime}$ and $\Lambda_{i}^{m}{ }^{\prime}$ denotes the exact and the modeled contributions of a given subgrid term (e.g. $C_{i}$ and $\left.C_{i}^{m}\right)$ :

$$
\begin{aligned}
\Lambda_{i}^{\prime} & =\frac{\partial \tau_{i j}}{\partial x_{j}}, \\
\Lambda_{i}^{m}{ }^{\prime} & =\frac{\partial \tau_{i j}^{m}}{\partial x_{j}} .
\end{aligned}
$$

We are thus seeking the model that minimises the dispersion error, $\varepsilon$. Relation number 43) gives an absolute error, but it does not inform about the relative proportions between exact and modeled term. We then propose to define three relative errors, considering the fact that each subgrid term contribution $\Lambda_{i}^{\prime}$, results from the total unclosed subgrid correlations $\Lambda_{c_{i}}$, subtracted by the resolved part $\Lambda_{r_{i}}$ :

$$
\begin{aligned}
\Lambda_{i}^{\prime} & =\Lambda_{c_{i}}-\Lambda_{r_{i}} \text { with } \\
\Lambda_{c_{i}} & =\frac{\partial \overline{\alpha_{i} \beta_{j}}}{\partial x_{j}} \\
\Lambda_{r_{i}} & =\frac{\partial \bar{\alpha}_{i} \bar{\beta}_{j}}{\partial x_{j}}
\end{aligned}
$$

where $\Lambda_{i}$ can be written in the following form: 


$$
\Lambda_{i}^{\prime}=\frac{\partial \tau_{i j}}{\partial x_{j}}=\frac{\partial \overline{\alpha_{i} \beta_{j}}-\bar{\alpha}_{i} \bar{\beta}_{j}}{\partial x_{j}} .
$$

In this last relation $\alpha$ and $\beta$ may represent any property of the flow. All the terms of decomposition in equation 46 are summed up in table 2.

Starting from this definition, we can obtain several relative errors, denoted $\varepsilon_{\text {sgs }}$, $\varepsilon_{\text {err }}$ and $\varepsilon_{\text {rap }}$ :

- $\varepsilon_{s g s}=\sigma\left(\Lambda_{i}^{\prime}\right) / \sigma\left(\Lambda_{c_{i}}\right)$ :

Strictly speaking, $\varepsilon_{s g s}$ is not an error, but indicates the proportion of the subgrid contribution $\Lambda_{i}^{\prime}$ compared to the total unclosed term $\Lambda_{c_{i}}$. It is expected that this proportion increases with filter size, $\bar{\Delta}$.

- $\varepsilon_{e r r}=\sigma\left(\Lambda_{i}^{\prime}-\Lambda_{i}^{m}{ }^{\prime}\right) / \sigma\left(\Lambda_{c_{i}}\right)$ :

$\varepsilon_{\text {err }}$ represents the proportion of the error made by the model compared to the subgrid correlation $\Lambda_{c_{i}}$. Thus, for an ideal model this error should tend to 0 .

- $\varepsilon_{r a p}=\varepsilon_{e r r} / \varepsilon_{\text {sgs }}=\sigma\left(\Lambda_{i}^{\prime}-\Lambda_{i}^{m}{ }^{\prime}\right) / \sigma\left(\Lambda_{i}^{\prime}\right)$

$\varepsilon_{\text {rap }}$ gives the ratio between the two last parameters. A value under $100 \%$ indicates that the model has a good behavior. On the contrary, a value over $100 \%$ indicates that the model overestimates, in a global sense, the subgrid contribution.

\subsubsection{Dispersion results}

The multiple errors which have been obtained using the operator $\sigma$ are shown in figures 17, 18 and 19 for convective, temporal and interfacial contributions, respectively. Note the use of the following common symbols notation for each tested contribution, direction and dispersion errors: 
- The size of the symbol is related to the filter length (i.e. smaller SFS, bigger LFS)

- Empty and filled symbols are related to the streamwise and spanwise contribution direction, respectively (except for the interfacial term, which is not directional).

- $\varepsilon_{s g s}$ is always represented with diamond symbols $(\diamond)$.

Firstly, we are interested in the dispersion errors made by the various models of convective term contribution ( figure 17).

The largest is the filter length, the highest $\varepsilon_{s g s}$ is (fig. 17-(a)), and this for both streamwise and spanwise directions. For example, in the streamwise direction in zone $3, \varepsilon_{\text {sgs }}$ start from about $10 \%$ with SFS and up to about $20 \%$ with LFS. This remark is also true for the temporal and convective contribution. It can be observed in figure 18-(a) and 19-(a). This result was expected, since there is more unresolved subgrid scales as the filter length increases.

In the streamwise direction, the convective SGS contribution is smaller in zone 3 than in zone 1 (fig. 17-(a)). Surprisingly, the opposite behavior can be observed for the spanwise direction contributions. Since we study the divergence of convective subgrid tensor in the $i^{\text {th }}$ direction, $C_{i}=\partial \tau_{\rho u_{i} u_{j}} / \partial x_{j}$, this behavior could be due to the isotropization of the velocity field in zone 3 at the small scales. Nevertheless, there is no clear explanation of this fact and more advanced study is required to advance the understanding of this troublesome point.

Scrutinizing $\varepsilon_{e r r}$ and $\varepsilon_{r a p}$, in figures 17-(b) and 17-(c), reveals the fact that the Smagorinsky model overestimates the subgrid contribution, for both constant and dynamic procedure. 
Nevertheless, this model leads to lower errors in zone 3 than in zone 1 (globally, $\varepsilon_{\text {rap }}>100 \%$ in zone 1 and $<100 \%$ in zone 3 ). These facts can be partially explained by the homogenization of the velocity field in zone 3 . However, unlike in zone 1 , we found an inertial range in the kinetic energy spectrum in zone 3 that is absent in zone 1 (c.f. figure 6). This fact can explain the bad results of the Smagorinsky procedure in zone 1. Nevertheless, the presence of an inertial range is implicitly considered in the construction of the Smagorinsky model. However, we notice that in zone 3 the dynamic procedure leads to slightly better results than the constant determination of $C_{s}$.

Finally, concerning the scale similarity closure model, figures 17-(b) and 17-(c) show that it offers better results than Smagorinsky models, in all considered cases. Indeed, $\varepsilon_{\text {rap }}$ is systematically under $100 \%$. Moreover, the quality of this closure is less affected by the considered direction or zone than the Smagorinsky model. For example in the SFS case, $\varepsilon_{\text {rap }}$ is close to $73 \%$ in zone 1 and 3 for streamwise contributions. For these last terms, in the LFS case, we found $79 \%$ and $85 \%$ for zone 1 and 3, respectively. Therefore, the filter size does not affect the model accuracy.

Thus, the scale similarity model seems to be much more versatile and it permits to obtain better estimations of subgrid contributions than the Smagorinsky models. However, we have to keep in mind that the scale similarity constant is optimised for each case thanks to the DNS.

Starting from this conclusion, we now consider errors obtained by the scale similarity model for the temporal contribution $T_{i}$, in figure 18 . The behavior of $\varepsilon_{\text {sgs }}$ for this term is fully similar to the one obtained for convective contribution.

The scale similarity closure gives an error $\varepsilon_{\text {rap }}$ under $100 \%$ in all tested cases. For all directions, this error is about $70 \%$ for SFS cases and about $80 \%$ in LFS cases. 
The last tested term is the interfacial contribution. Note that there is no direction aspect since we observe the dispersion errors for $\Pi=\overline{u_{i} \partial \chi} / \partial x_{i}-\bar{u}_{i} \overline{\partial \chi} / \partial x_{i}$. The results obtained for this term are plotted in figure 19. Firstly, the study of $\varepsilon_{s g s}$ reveals that the proportion of subgrid contributions to close becomes more important with increasing filter size, in the same manner as the other two contributions. We can also notice, the fact that this proportion is bigger in zone 1 than in zone 3 . Again, the scale similarity model permits to obtain dispersion error, $\varepsilon_{\text {err }}$ and $\varepsilon_{\text {rap }}$, values under $100 \%$ for each tested zone.

The study of the dispersion errors obtained in this section clearly shows that the scale similarity model is more able to reproduce the convective subgrid contribution of transport equations than the usual Smagorinsky model, even with dynamic procedure. Once the constant $C_{c}, C_{t}$ and $C_{\chi}$ are correctly determined, the application of this model on temporal and interfacial terms also shows good behaviors. From this point of view, the conclusions here are in full agreement with other a priori studies ([Boivin et al., 2000; Toutant et al., 2008]) in different two-phase flow configurations. These studies suggest that the scale similarity model or mixed model, which include this assumption, lead to better estimations of subgrid contributions than the classical eddy viscosity models. We thus reach the same conclusion for a liquid/gas flow in an atomization regime.

Nevertheless, we can recall the fact that a priori testing, in the way it has been led, implies some drawbacks. On the one hand, the validity of the results obtained here can only be assumed for the tested Reynolds number, which is relatively low. The quality of the subgrid closure has to be confirmed for higher Reynolds or Weber numbers. On the other hand, the high computational of DNS did not permit to take into account the largest time scale interactions between the subgrid terms. Consequently, a possible accumulation of errors made by a closure on long time 
simulations cannot be detected with this sort of a priori test.

Assuming an accumulation of the errors, we notice that the dispersion errors found even with the scale similarity model are somewhat higher (more than 50\% for $\left.\varepsilon_{\text {sgs }}\right)$. Moreover, the gain obtained when applying the scale similarity model by comparison to directly neglect the subgrid terms is quite low. In fact, the mean error $\varepsilon_{\text {rap }}$ range lies between $60 \%$ and $80 \%$. Thus, it could be interesting to test other subgrid models, like the mixed model [Bardina et al., 1980], in order to know if it is possible to obtain better results.

\section{Conclusions}

In this paper, the direct numerical simulation of a fully atomizing jet of liquid has been carried out to evaluate, from an a priori point of view, the various subgrid properties from the liquid destabilization area, close to the injector, down to the fully atomized jet. Simulations have been performed thanks to the coupling of level-set and VOF formulations.

An a priori analysis of the subgrid terms has been carried out to evaluate the order of magnitude of the contributions of these terms to the flow evolution. It is the first time that this kind of analysis is carried out on such a complex configuration. The results show that, wherever we are positioned in the atomizing jet, the hierarchy between the subgrid terms of the momentum equation remains the same with a prevalence of the temporal and the convective terms. This observation confirms previous works carried out on simpler configurations. The evolution equation of the filtered composition field has been scrutinized as well. It appears that the interface evolution is not mainly controlled by large-scale convection phenomena. Thus, it is necessary to take into account the impact of the small scales on the interface propagation. Furthermore, at the interface level close to the injection, the order of 
magnitude of the subgrid correlations increases strongly. Knowing that the initial liquid destabilization process could appear at a subgrid level, it may be dangerous to neglect them. Therefore a closure has been proposed and evaluated as well. The choice has been made to use a scale similarity assumption to close all the subgrid unresolved terms. Classical dynamic and static Smagorinsky formulations have been tested as well, but the results were not satisfactory at all because of the presence of the interface that leads to strong gradients, which are not related to the turbulent motion. The scale similarity assumption gave better results as far as the DNS of an atomizing jet is concerned. This means that the geometry dimensions remain small with a liquid Reynolds number equal to 5800. One of the main encouraging conclusions is the fact that the models are able to capture the subgrid terms wherever we are in the jet: in the atomization area or far from the injector.

To go further, a priori tests have been extended by the analysis of the statistical dispersion of the error made by the various models. The choice has been made to consider a possible accumulation of the error. The clear benefit to using the scale similarity model by comparison with the eddy viscosity model has been demonstrated. However, even if the scale similarity model brings systematically an improvement, errors due to the model remain. This implies that more efforts should be necessary to improve subgrid atomization modeling for LES of atomization.

Very encouraging results have been observed and the next stage is now to carry out effective LES with a posteriori validations and the development of a Eulerian procedure to describe the dispersion of the droplets reaching a subgrid size.

\section{Acknowledgement}

This work was granted access to the HPC resources of IDRIS under the allocation 2010-x2010026153 made by GENCI (Grand Equipement National de Calcul Intensif). 
The authors also acknowledge the computational support of CRIHAN (Centre de Ressources Informatiques de Haute-Normandie) and Renault S.A. for their financial support. 


\section{References}

Bardina, J., Ferziger, J., Reynolds, W., 1980. Improved subgrid-scale models for large-eddy simulation. AIAA paper-80.

Baumgarten, C., 2006. Mixture formation in Internal Combustion Engine. Springer.

Berlemont, A., Tanguy, S., 2005. Development of a level set method for interface tracking : applications to droplet collisions. Int. J. Multiphase Flows 31, 10151035.

Bianchi, G., Minelli, F., Scardovelli, R., Zaleski, S., 2007. 3d large scale simulation of the high-speed liquid jet atomization. SAE international2007-01-0244.

Boivin, M., Simonin, O., Squires, K. D., 2000. On the prediction of gas-solid flows with two-way coupling using large eddy simulation. Physics of Fluids 12 (8), 20802090.

Brackbill, J., Khote, D., Zemach, C., 1992. A continuum method for modelling surface tension. J. Comput. Phys. 100, 335-354.

Buonfiglioli, M., Mendonca, F., 2005. LES-VOF simulation of primary diesel spray break-up with synthetic inlet perturbations. In: ILASS america.

Chesnel, J., Ménard, T., Réveillon, J., Berlemont, A., Demoulin, F.-X., 2010. A les simulation of atomisation. In: ASME, Montreal. No. FEDSM-ICNMM2010-30664.

Chesnel, J., Réveillon, J., Demoulin, F. X., Ménard, T., 2007. Subgrid modeling of liquid atomization. In: 6th International Conference on Multiphase Flow.

Christensen, E. D., 2006. Large eddy simulation of spilling and plunging breakers. Coastal Engineering 53 (5-6), $463-485$. 
De Villier, E., Gosman, A., Weller, H., 2004. Large eddy simulation of primary diesel spray atomisation. SAE international 113 (3), 193-206.

Deen, N., Solberg, T., Hjertager, B., 2001. Large eddy simulation of the gas-liquid flow in a square cross-sectionned bubble column. Chemical Engineering science 5, 6341-6349.

Delhaye, J. M., 1974. Jump conditions and entropy sources in two-phase systems. local instant formulation. International Journal of Multiphase Flow 1 (3), 395 409.

Demoulin, F., Beau, P., Blokkeel, G., Mura, A., Borghi, R., 2007. A new model for turbulent flows with large density fluctuations: application to liquid atomization. Atom. and Sprays 17, 315-345.

Drew, D., 1983. Mathematical modeling of two phase flow. Ann. Rev. Fluid Mech. $15,261-291$.

Faeth, G. M., 1991. Structure and atomization properties of dense turbulent sprays. In: Symposium (International) on Combustion, 23rd, Orleans, France, July 2227, 1990, Proceedings (A92-16529 04-25). Pittsburgh, PA, Combustion Institute, 1991, p. 1345-1352. pp. 1345-1352.

Fedkiw, R. P., Aslam, T., Merriman, B., Osher, S., 1999. A non-oscillatory eulerian approach to interfaces in multimaterial flows (the ghost fluid method). Journal of Computational Physics 152 (2), 457 - 492.

Germano, M., 1992. Turbulence: the filtering approach. J. Fluid Mech. 238, 325-336.

Germano, M., Piomelli, U., Moin, P., Cabot, W., 1991. A dynamic subgride-scale eddy viscosity model. Phy. Fluids 7(3), 1760-1765. 
Herrmann, M., Gorokhovski, M., 2008. An outline of a les subgrid model for liquid/gas phase interface dynamics. In: Proceedings of the 2008 CTR Summer Program, Center for Turbulence Research, Stanford University, Stanford, CA. pp. $171-181$.

Hieu, P., Katsutoshi, T., Ca, V., 2004. Numerical simulation of breaking waves using a two phase flow model. appl. Math. Model. 28, 983-1005.

Hirt, C., Nichols, B., 1981. Volume of fluid (vof) - method for the dynamics of free boundaries. J. Comput. Phys. 39, 201-225.

Kang, M., Liu, X., Fedkiw, R., 2000. Boundary condition capturing method for multiphase imcompressible flows. J. Sci. Comput. 15, 323-360.

Kataoka, I., 1986. Local instant formulation of two-phase flow. International Journal of Multiphase Flow 12 (5), 745 - 758.

Klein, M., Sadiki, A., Janicka, J., 2003. A digital filter based generation of inflow data for spatially developing direct numerical or large eddy simulations. Journal of Computational Physics 186 (2), $652-665$.

Labourasse, E., Lacanette, D., Toutant, A., Lubin, P., Vincent, S., Lebaigue, O., Caltagirone, J.-P., Sagaut, P., 2007. Towards large eddy simulation of isothermal two-phase flows: Governing equations and a priori tests. International Journal of Multiphase Flow 33 (1), 1 - 39 .

Lakehal, D., Meier, M., Fulgosi, M., 2002. Interface tracking towards the direct simulation of heat and mass transfer in multiphase flows. International Journal of Heat and Fluid Flow 23 (3), $242-257$. 
Larocque, J., Vincent, S., Lacanette, D., Lubin, P., Caltagirone, J.-P., 2010. Parametric study of les subgrid terms in a turbulent phase separation flow. International Journal of Heat and Fluid Flow In Press, Corrected Proof,-.

Law, C., 2006. Combustion Physics. Cambridge University Press.

Lebas, R., Menard, T., Beau, P., Berlemont, A., Demoulin, F., 2009. Numerical simulation of primary break-up and atomization: DNS and modelling study. International Journal of Multiphase Flow 35 (3), 247 - 260.

Lee, S., Lele, K., Moin, P., 1991. Numerical simulations of spatially evolving compressible turbulence. Center for Turbulence Research, Annual Research Briefs, Stanford 126.

Lilly, D., 1992. A proposed modification of the germano subgridscale closure method. Phys. Fluid A 4, 633-635.

Liovic, P., Lakehal, D., 2007. Interface-turbulence interactions in large-scale bubbling processes. International Journal of Heat and Fluid Flow 28 (1), 127 - 144, the International Conference on Heat Transfer and Fluid Flow in Microscale (HTFFM05), The International Conference on Heat Transfer and Fluid Flow in Microscale.

Lubin, P., Vincent, S., Abadie, S., Caltagirone, J.-P., 2006. Three-dimensional large eddy simulation of air entrainment under plunging breaking waves. Coastal Engineering $53(8), 631-655$.

Menard, T., Tanguy, S., Berlemont, A., 2007. Coupling level set/vof/ghost fluid methods: Validation and application to $3 \mathrm{~d}$ simulation of the primary break-up of a liquid jet. International Journal of Multiphase Flow 33, 510-524. 
Nicoud, F., Ducros, F., 1999. Subgrid-scale stress modelling based on the square of the velocity gradient tensor. Flow, Turbulence and Combustion 62, 183-200(18).

Orszag, S., Patterson, G., Jan 1972. Numerical simulation of three-dimensional homogeneous isotropic turbulence. Phys. Rev. Lett. 28 (2), 76-79.

Osher, S., Sethian, J., 1988. Fronts propagating with curvature-dependent speed: Algorithms based on hamilton-jacobi formulations. Journal of Computational Physics $79(1), 12-49$.

Reitz, R. D., 1978. Atomization and other break-up regimes of a liquid jet. Ph.D. thesis, Princeton University.

Rogallo, R. S., 1981. Numerical experiments in homogeneous turbulence. Tech. rep., NASA, technical Memorandum No. 81315.

Sagaut, P., 2003. Large Eddy Simulation for Imcompressible Flows. Springer Verlag.

Sagaut, P., Germano, M., 2005. On the filtering paradigm for les of flows with discontinuities. Journal of Turbulence 6, N23.

Sagaut, P., Montreuil, E., Labb, O., 1999. Assessment of some self-adaptive sgs models for wall bounded flows. Aerospace Science and Technology 3 (6), 335 344.

Sirignano, W. A., 1983. Fuel droplet vaporization and spray combustion theory. Progress in Energy and Combustion Science 9, 291-322.

Smagorinsky, J., 1963. General experiments with the primitives equations. Mon. Weather Rev. 63, 99-164. 
Sussman, M., Puckett, E. G., 2000. A coupled level set and volume-of-fluid method for computing $3 \mathrm{~d}$ and axisymmetric incompressible two-phase flows. Journal of Computational Physics 162 (2), $301-337$.

Tanguy, S., T., M., Berlemont, A., 2007. A level set method for vaporizing two-phase flows. Journal of Computational Physics 221 (2), 837 - 853.

Toutant, A., Labourasse, E., Lebaigue, O., Simonin, O., 2008. DNS of the interaction between a deformable buoyant bubble and a spatially decaying turbulence: A priori tests for les two-phase flow modelling. Computers and Fluids 37 (7), 877 - 886, special Issue of the "Turbulence and Interaction-TI2006" Conference.

Vallet, A., Burluka, A., Borghi, R., 2001. Developpement of an eulerian model for the atomization of a liquid jet. Atom. and Sprays 11, 619-642.

van der Pijl, S. P., Segal, A., Vuik, C., Wesseling, P., 2005. A mass-conserving levelset method for modelling of multi-phase flows. International Journal for Numerical Methods in Fluids 47, 339-361.

Vincent, S., Larocque, J., Lacanette, D., Toutant, A., Lubin, P., Sagaut, P., 2008. Numerical simulation of phase separation and a priori two-phase les filtering. Computers and Fluids 37 (7), 898 - 906, special Issue of the "Turbulence and Interaction-TI2006" Conference.

Wang, P., Frhlich, J., Michelassi, V., Rodi, W., 2008. Large-eddy simulation of variable-density turbulent axisymmetric jets. International Journal of Heat and Fluid Flow 29 (3), 654 - 664, the Fifth International Symposium on Turbulence and Shear Flow Phenomena (TSFP5). 
Yue, W., Lin, C.-L., Patel, V. C., 2005. Large eddy simulation of turbulent openchannel flow with free surface simulated by level set method. Physics of Fluids $17(2), 025108$.

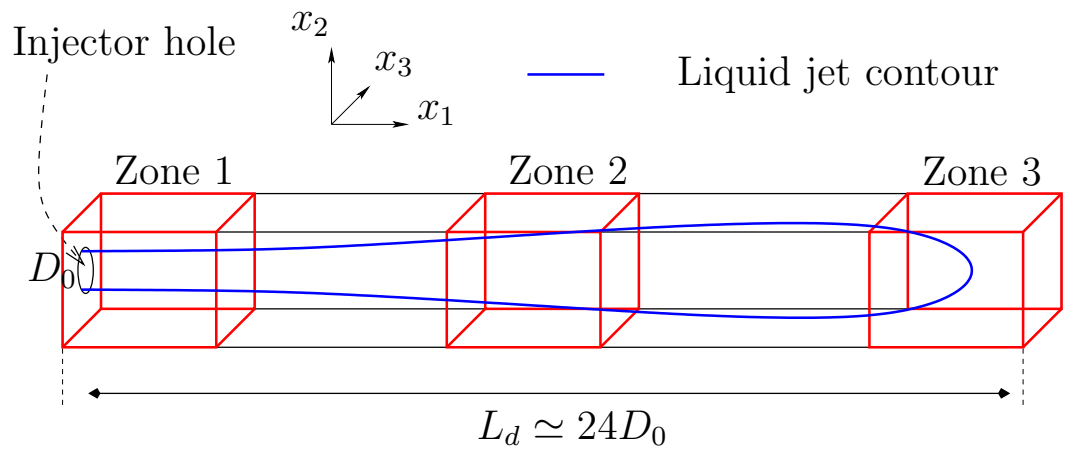

Figure 1: Computational domain. 

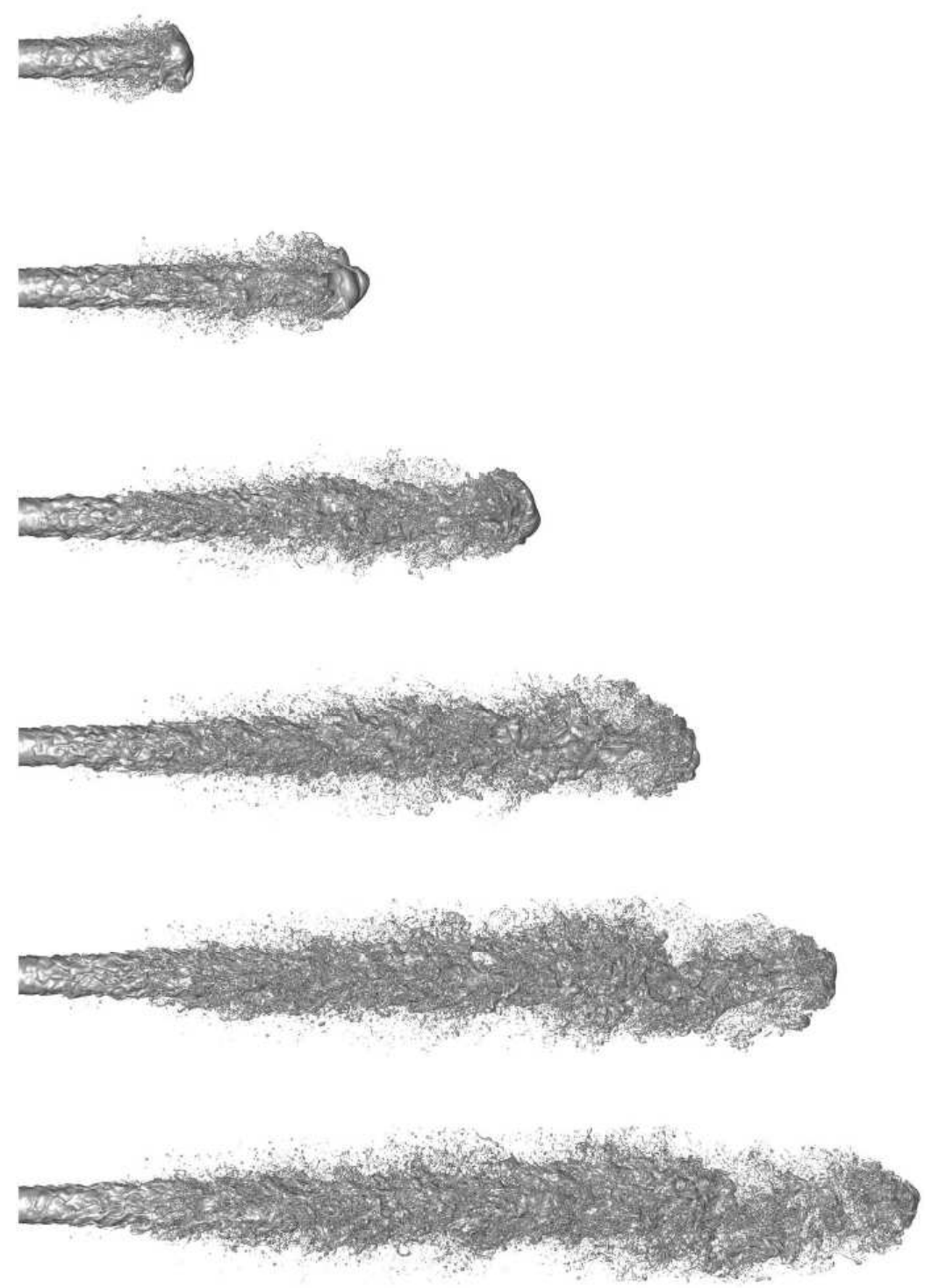

Figure 2: DNS of a liquid jet atomization 


\begin{tabular}{|l|l|l|}
\hline Parameter & Symbol/Unit & Value \\
\hline \hline Gas density & $\rho_{g}\left(\mathrm{~kg} \cdot \mathrm{m}^{-3}\right)$ & 25 \\
\hline Liquid density & $\rho_{l}\left(\mathrm{~kg} \cdot \mathrm{m}^{-3}\right)$ & 696 \\
\hline Gas viscosity & $\mu_{g}\left(\mathrm{~kg} \cdot \mathrm{m}^{-1} \mathrm{~s}^{-1}\right)$ & $1 \cdot \mathrm{x} 10^{-5}$ \\
\hline Liquid viscosity & $\mu_{l}\left(\mathrm{~kg} \cdot \mathrm{m}^{-1} \mathrm{~s}^{-1}\right)$ & $1.18 \mathrm{x} 10^{-3}$ \\
\hline Surface tension coefficient & $\sigma\left(N \cdot \mathrm{m}^{-1}\right)$ & 0.06 \\
\hline Injection Diameter & $D_{0}\left(\mu m^{\prime}\right)$ & 100 \\
\hline Mean flow rate velocity & $U_{d}\left(m \cdot s^{-1}\right)$ & 79 \\
\hline Liquid Reynolds & $R e_{l}$ & 4659 \\
\hline Liquid Weber & $W e_{l}$ & 7239 \\
\hline Turbulence Intensity & $\overline{u^{\prime} u^{\prime}} / U^{2}$ & 0.05 \\
\hline Turbulent scale & $L_{t}(m)$ & $0.1 D_{0}$ \\
\hline
\end{tabular}

Table 1: Physical parameters 


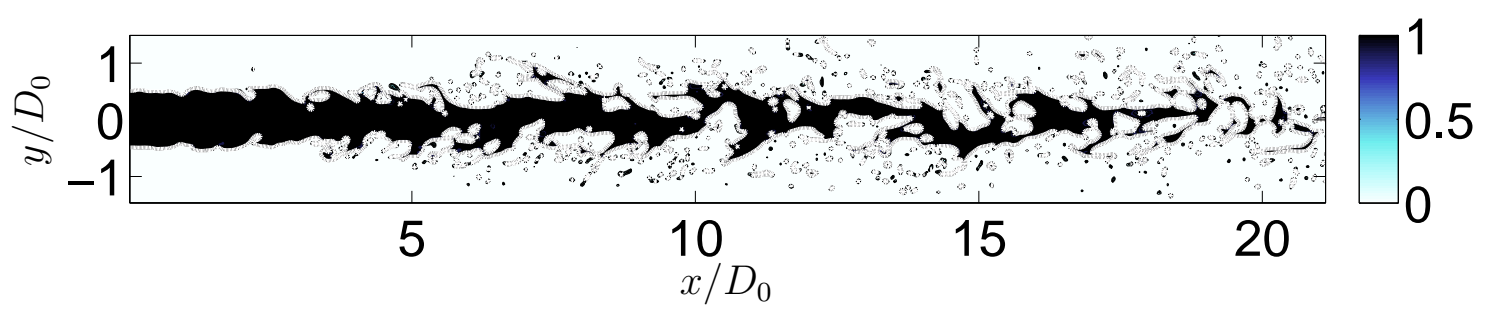

(a) DNS field

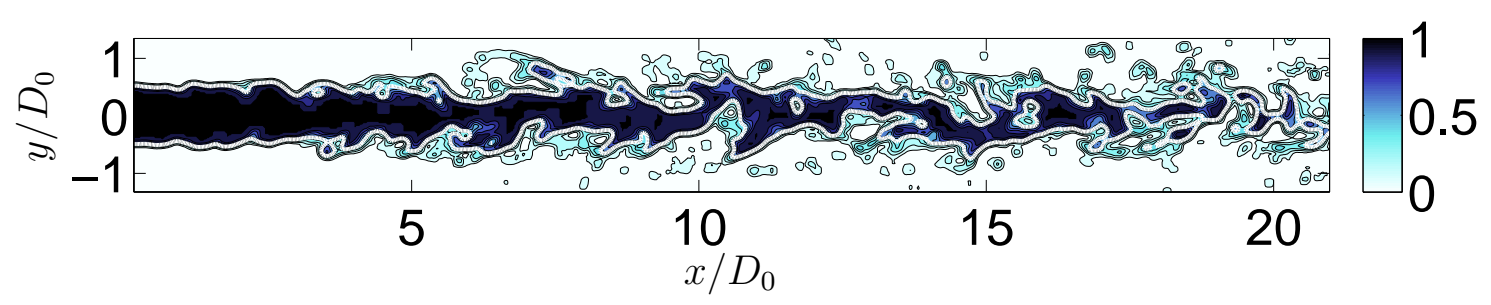

(b) $\bar{\Delta}=5 \Delta$.

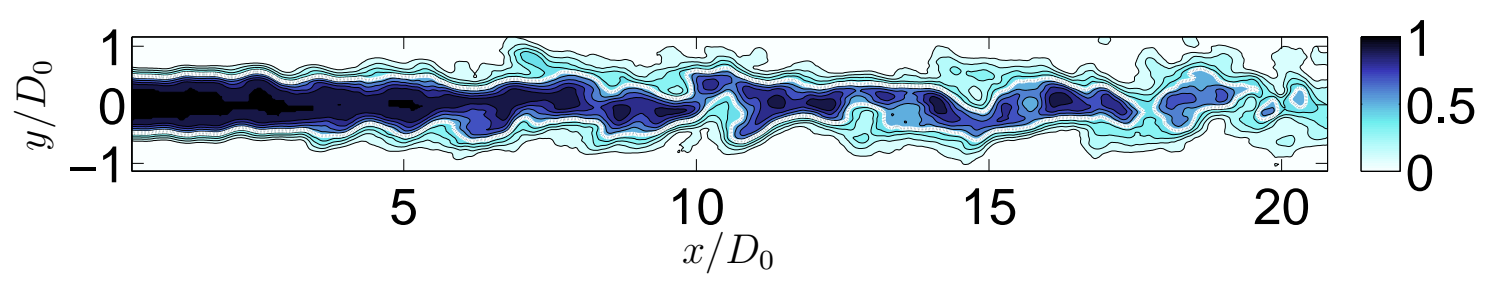

(c) $\bar{\Delta}=13 \Delta$.

Figure 3: Impact of filtering on the phase variable, streamwise direction $(x-z)$ cuts. The white line represents the interface position. (a) : DNS field, (b) : SFS $\bar{\Delta}=5 \Delta$, (c) : LFS $\bar{\Delta}=13 \Delta$. 


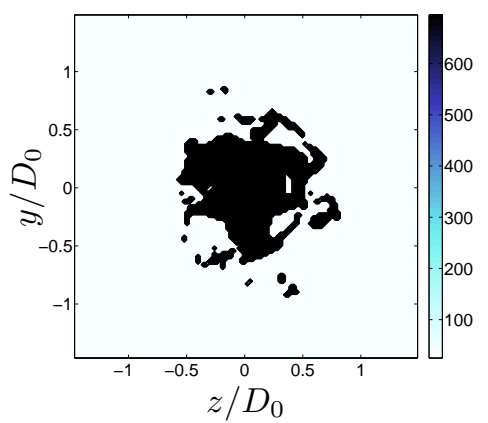

(a) $\rho$ DNS, zone 1 .

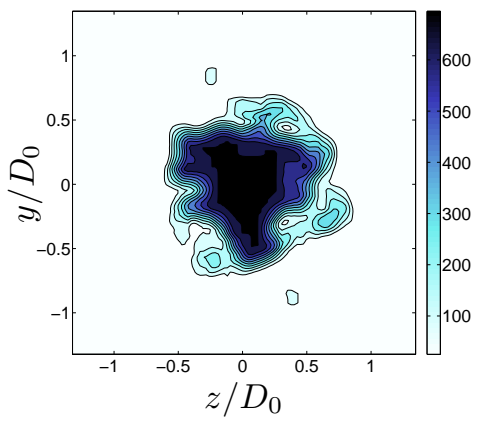

(d) $\bar{\rho}(\bar{\chi})$, zone 1 .

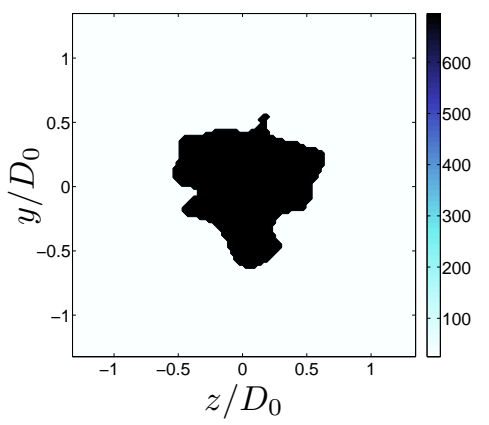

(g) $\bar{\rho}(\varepsilon)$, zone 1 .

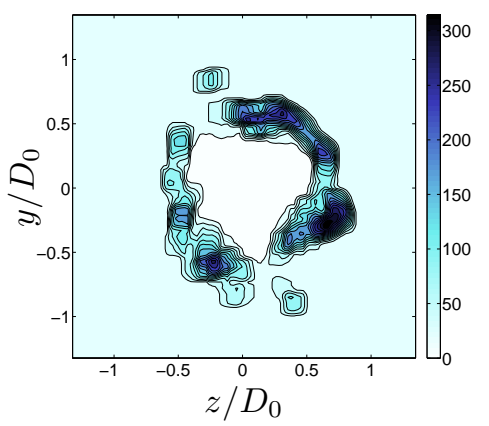

(j) $\rho(\varepsilon)$ subgrid, zone 1 .

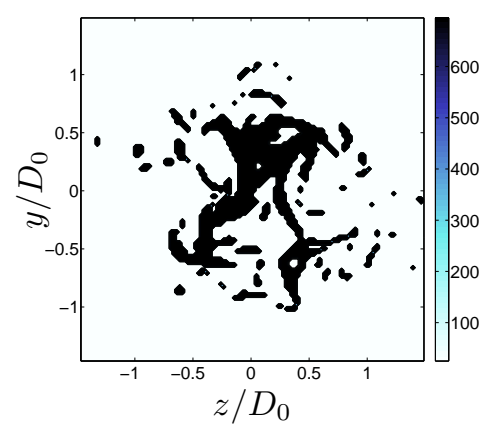

(b) $\rho$ DNS, zone 2 .

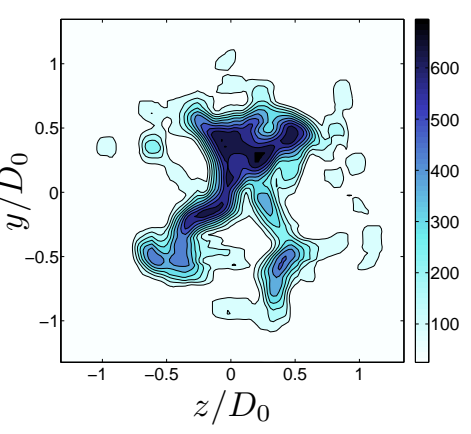

(e) $\bar{\rho}(\bar{\chi})$, zone 2 .

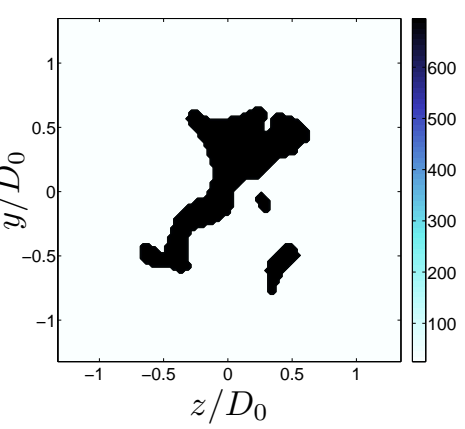

(h) $\bar{\rho}(\varepsilon)$, zone 2 .

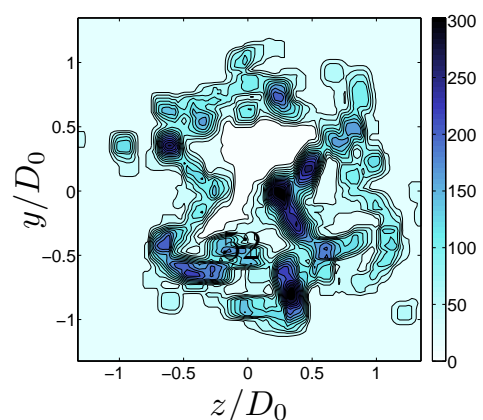

(k) $\rho(\varepsilon)$ subgrid, zone 2 .

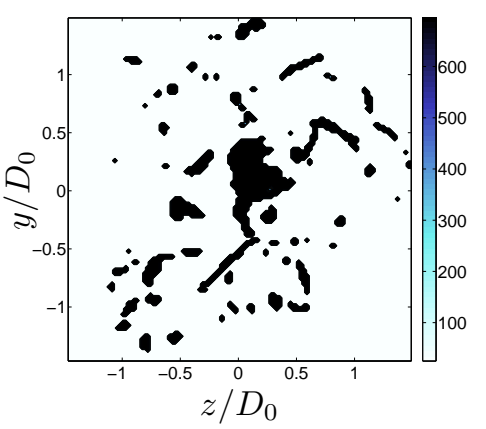

(c) $\rho$ DNS, zone 3 .

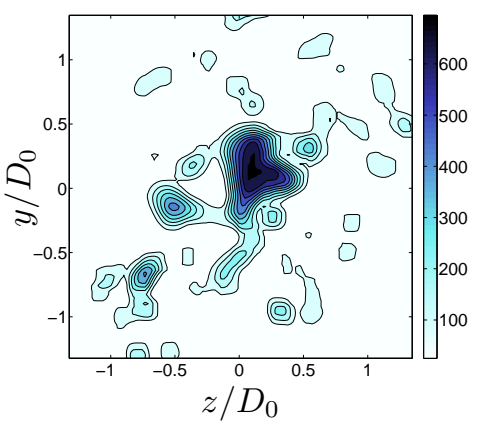

(f) $\bar{\rho}(\bar{\chi})$, zone 3 .

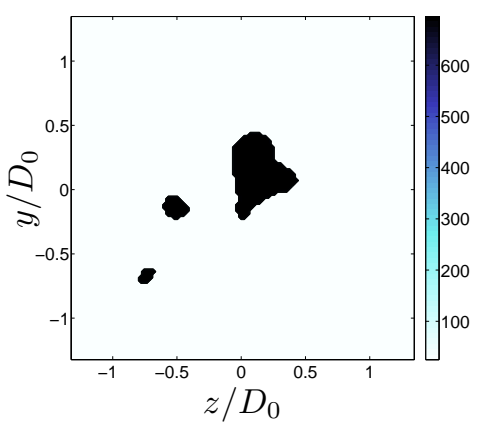

(i) $\bar{\rho}(\varepsilon)$, zone 3 .

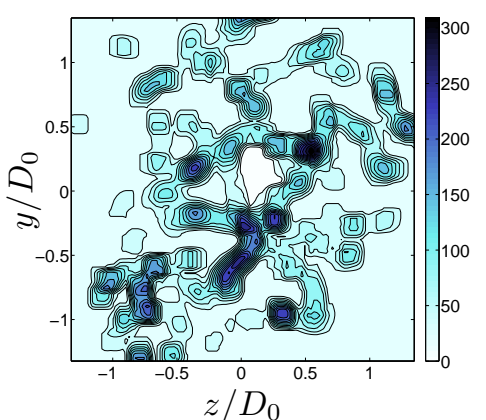

(l) $\rho(\varepsilon)$ subgrid, zone 3 .

Figure 4: Densities $\rho$ and $\bar{\rho}$ on spanwise cuts $x y$ for zones 1,2 and 3 . 


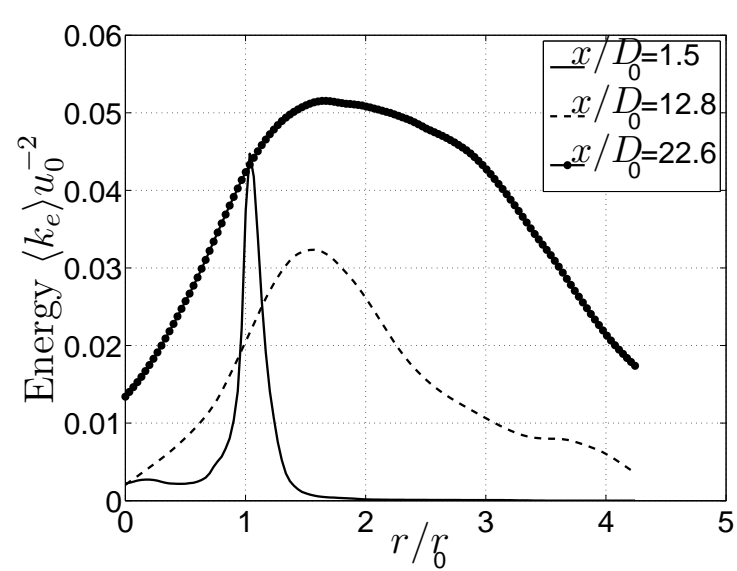

(a) Turbulent kinetic energy, $\left\langle k_{e}\right\rangle$

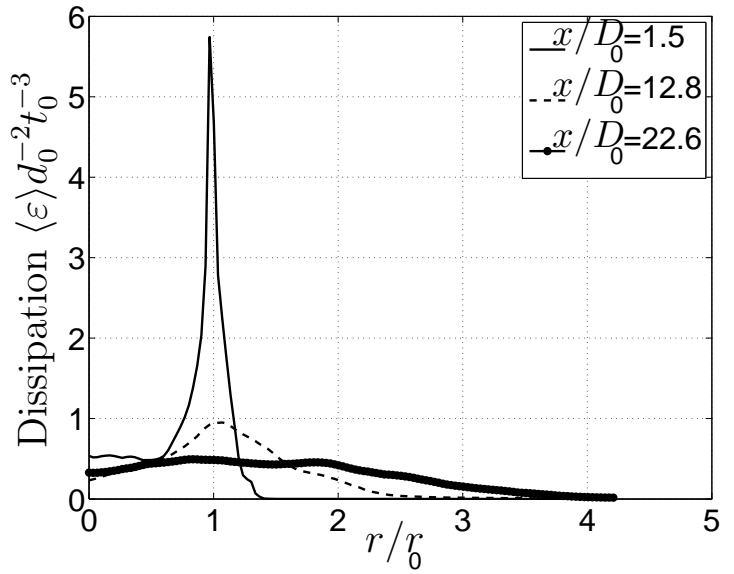

(b) Dissipation, $\langle\varepsilon\rangle$

Figure 5: Turbulence properties evolution along the streamwise direction. Three positions : $1.5,12.8$ and $22.6 D_{0}$.

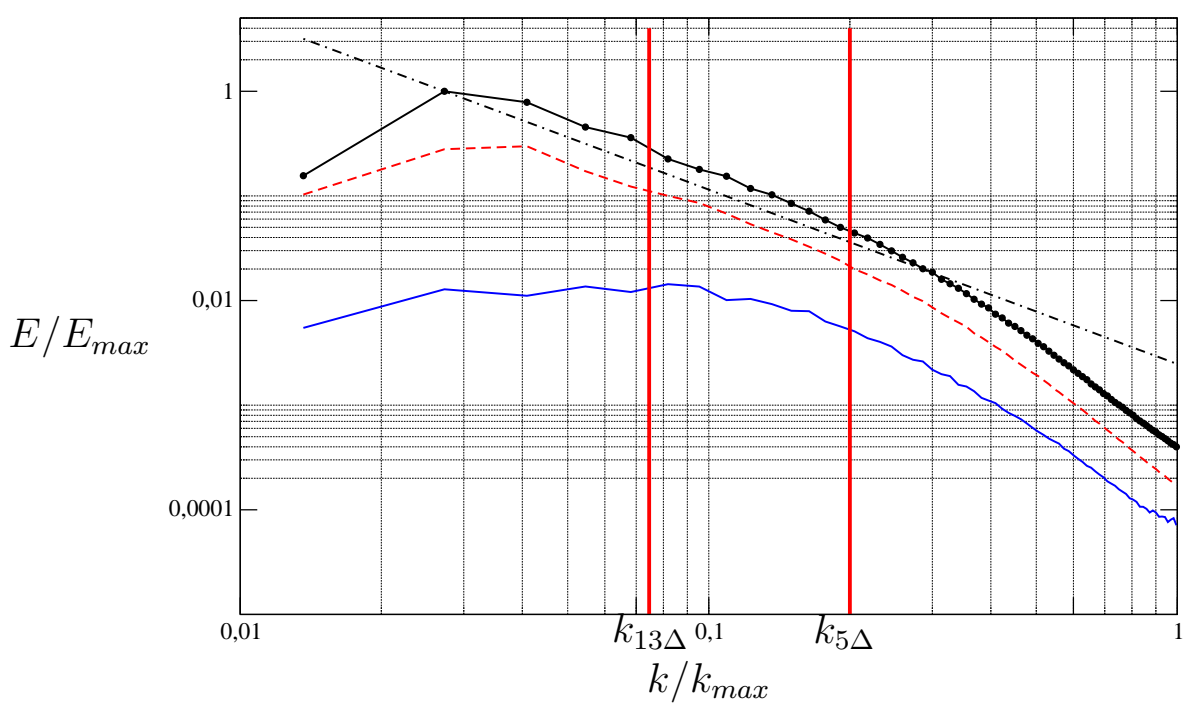

Figure 6: Kinetic energy spectrum. Dot-dashed straight line $: k^{-5 / 3}$, line with symbol : injection area $\left(0-4 D_{0}\right)$, dashed line : central area $\left(8-12 D_{0}\right)$, solid line : atomized area $\left(20-24 D_{0}\right)$. 


\begin{tabular}{|c|c|}
\hline SGS term & $\Lambda_{i}^{\prime}$ \\
\hline$\tau_{\rho u_{i} u_{j}}$ & $C_{i}^{\prime}=\partial\left(\overline{\rho u_{i} u_{j}}-\bar{\rho} \bar{u}_{i} \bar{u}_{j}\right) / \partial x_{j}$ \\
\hline$\tau_{\rho u_{i}}$ & $T_{i}^{\prime}=\partial\left(\overline{\rho u_{i}}-\bar{\rho} \bar{u}_{i}\right) / \partial t$ \\
\hline$\tau_{\chi}$ & $\Pi^{\prime}=\overline{u_{i} \partial \chi} / \partial x_{i}-\bar{u}_{i} \overline{\partial \chi} / \partial x_{i}$ \\
\hline \hline SGS term & $\Lambda_{c_{i}}$ \\
\hline$\tau_{\rho u_{i} u_{j}}$ & $C_{c_{i}}=\partial \overline{\rho u_{i} u_{j}} / \partial x_{j}$ \\
\hline$\tau_{\rho u_{i}}$ & $T_{c_{i}}=\partial \overline{\rho u_{i}} / \partial t$ \\
\hline$\tau_{\chi}$ & $\Pi_{c}=\overline{u_{i} \partial \chi} / \partial x_{i}$ \\
\hline \hline SGS term & $\Lambda_{r_{i}}$ \\
\hline$\tau_{\rho u_{i} u_{j}}$ & $C_{r_{i}}=\partial \bar{\rho} \bar{u}_{i} \bar{u}_{j} / \partial x_{j}$ \\
\hline$\tau_{\rho u_{i}}$ & $T_{r_{i}}=\partial \bar{\rho} \bar{u}_{i} / \partial t$ \\
\hline$\tau_{\chi}$ & $\Pi_{r}=\bar{u}_{i} \overline{\partial \chi} / \partial x_{i}$ \\
\hline
\end{tabular}

Table 2: Expressions of the resolved/unresolved decomposition terms (eq. 46) of the convective, temporal and interfacial contributions. 


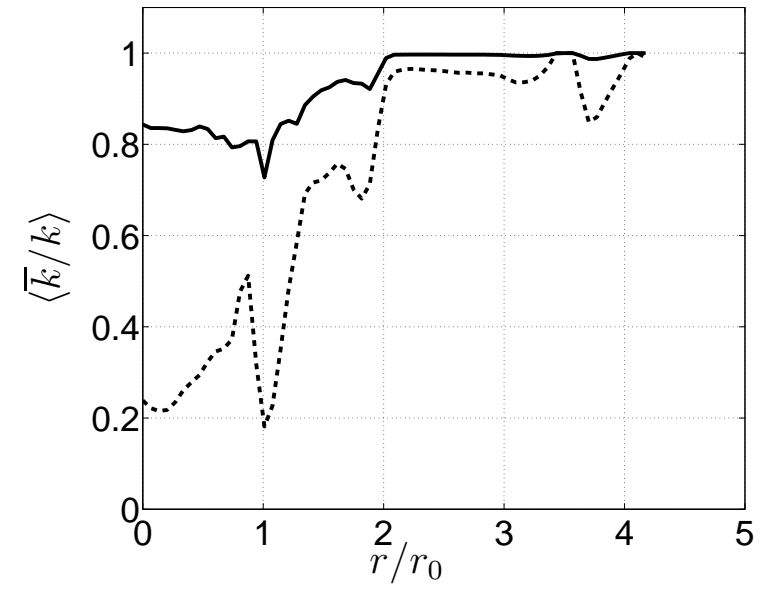

(a) Position $1.5 D_{0}$, zone 1 .

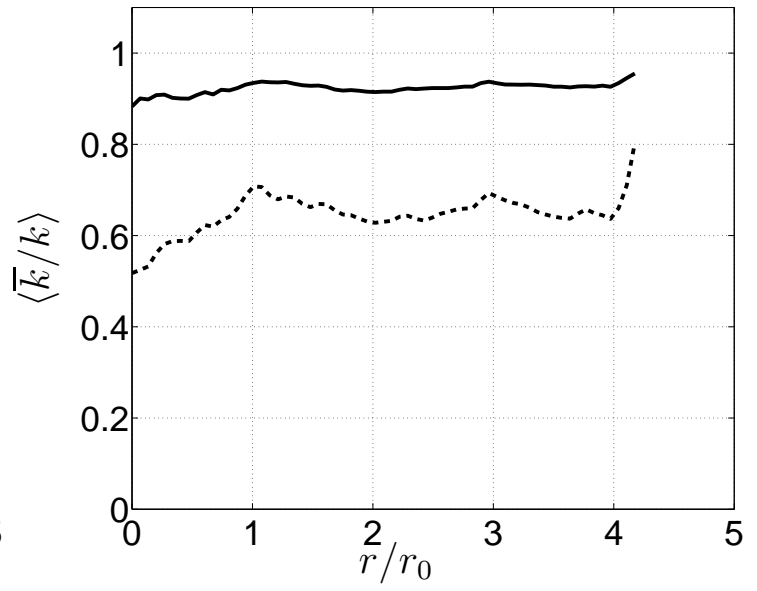

(b) $18.5 D_{0}$, zone 3 .

Figure 7: Ratio of the resolved kinetic energy $\bar{k}$ with the total kinetic energy $k$, radial mean. Solid lines : $\bar{\Delta}=5 \Delta$, dashed : $\bar{\Delta}=13 \Delta$. 


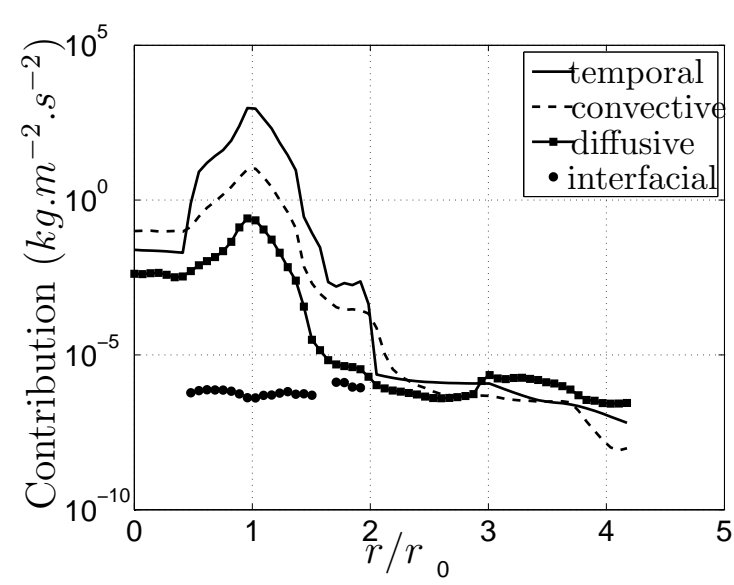

(a) Streamwise momentum $\rho u_{1}$, position : 5

$D_{0}$

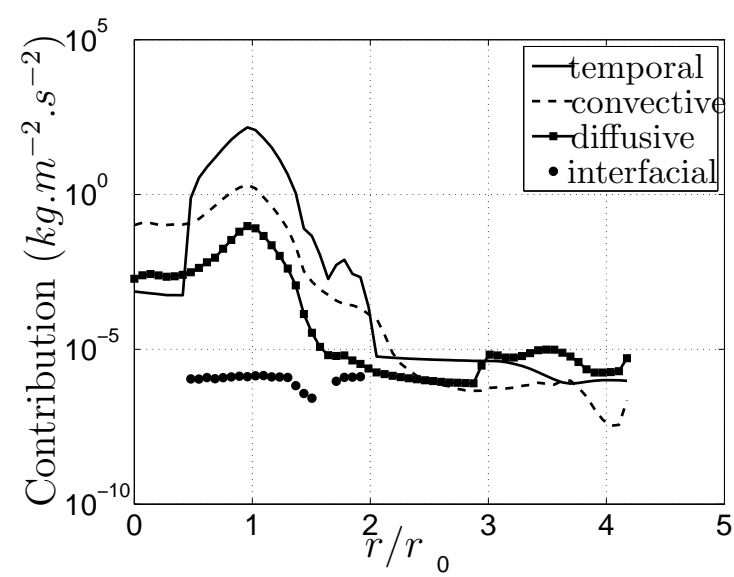

(c) Spanwise momentum $\rho u_{3}: 5 D_{0}$

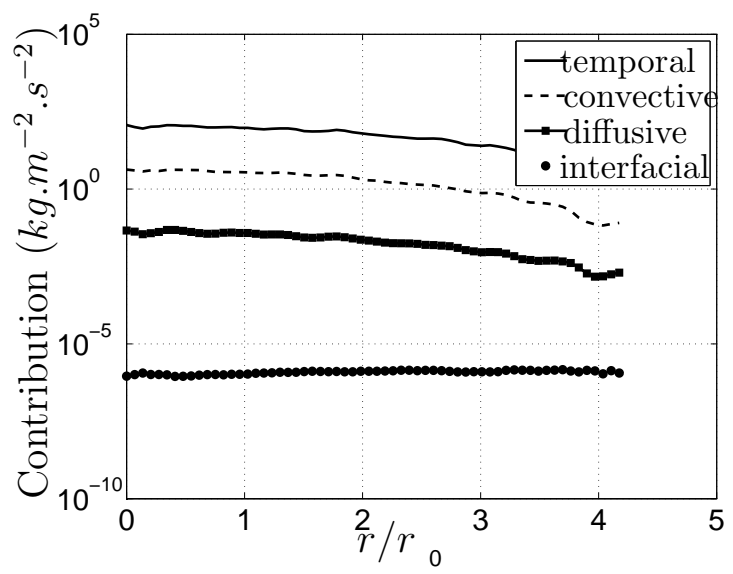

(b) Streamwise momentum $\rho u_{1}$, position 20 $D_{0}$

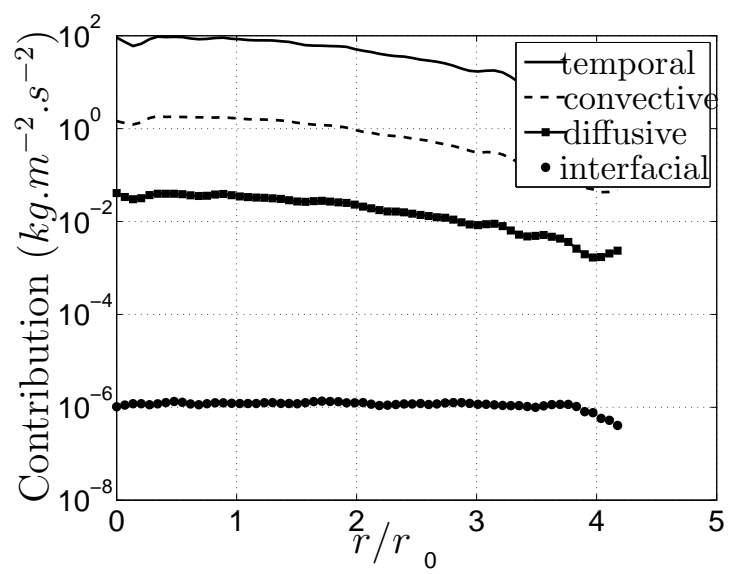

(d) Spanwise momentum $\rho u_{3}: 20 D_{0}$

Figure 8: Subgrid contributions for momentum equation. $\bar{\Delta}=5 \Delta . \log$ scale. 


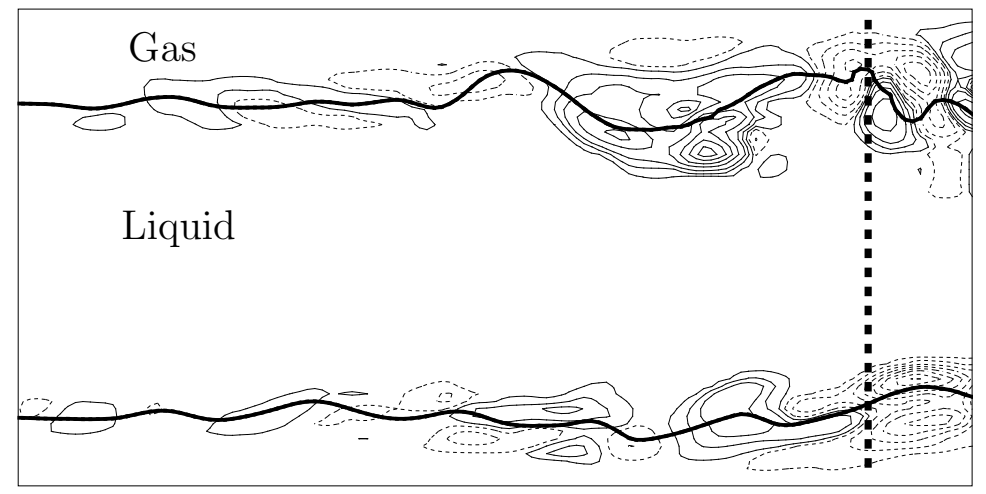

(a) $\tau_{\rho u_{1} u_{2}}, x y$ cut in zone 1 .

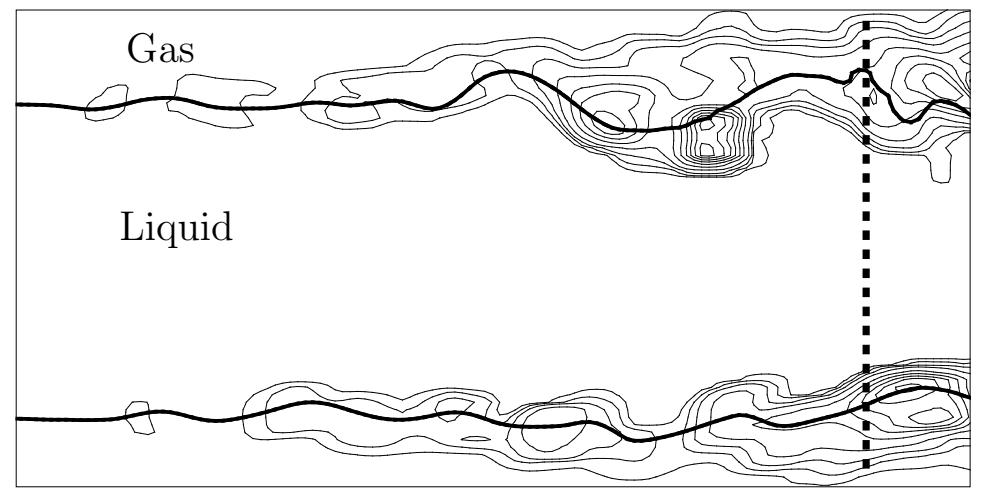

(c) $\tau_{\rho u_{2} u_{2}}, x y$ cut in zone 1 .

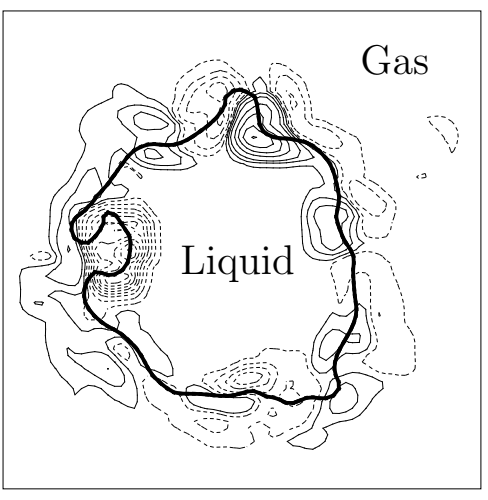

(b) $\tau_{\rho u_{1} u_{2}}, y z$ cut.

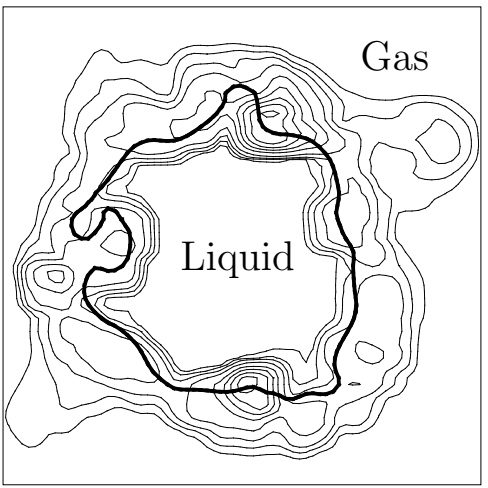

(d) $\tau_{\rho u_{2} u_{2}}, y z$ cut.

Figure 9: Thin lines : momentum subgrid contribution estimation (plain lines : $\tau>0$, dashed lines $\tau<0$ ), LFS case. Thick line : smoothed interface contour. Minimum and maximum values for $\tau_{\rho u_{1} u_{2}}$ are $-1.6 \times 10^{5}$ and $2.4 \times 10^{5} \mathrm{~kg} \cdot \mathrm{m}^{-2} . \mathrm{s}^{-2}$, respectively. Minimum and maximum values for $\tau_{\rho u_{2} u_{2}}$ are $2 \times 10^{3}$ and $2 \times 10^{5} \mathrm{~kg} \cdot \mathrm{m}^{-2} . \mathrm{s}^{-2}$, respectively. 


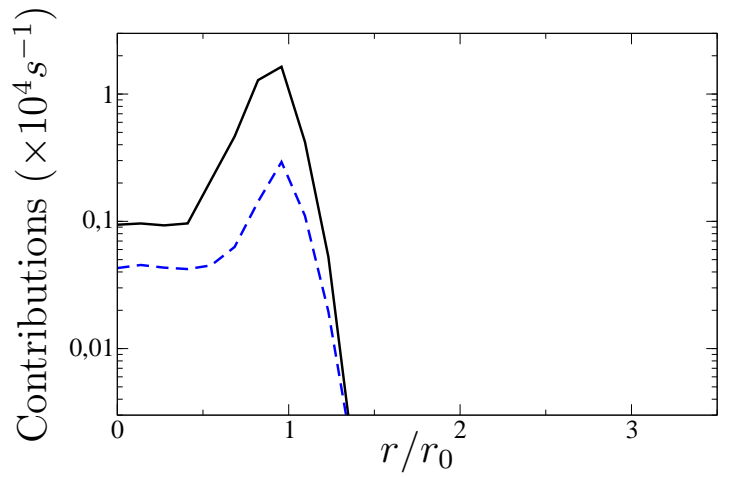

(a) Zone 1 .

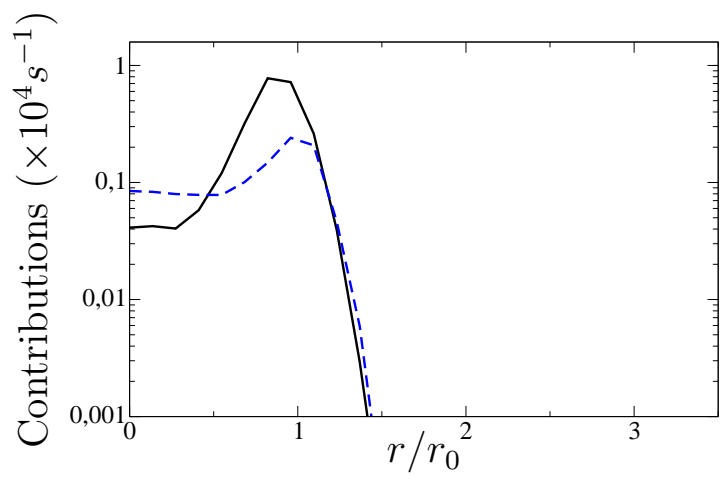

(c) Zone 1 .

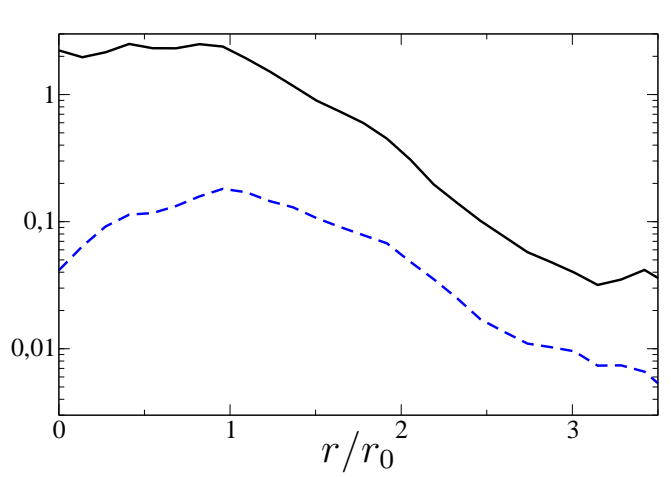

(b) Zone 3 .

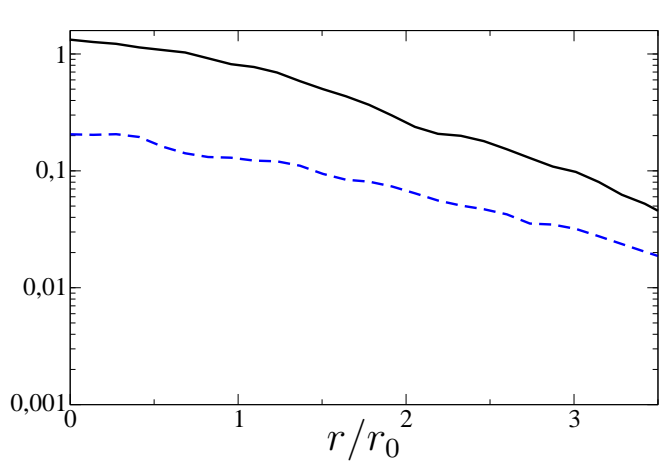

(d) Zone 3 .

Figure 10: Resolved (solid lines) and subgrid (dashed lines) contributions to the phase function evolution equation. Zone 1 (a) and 3 (b) : $\bar{\Delta}=5 \Delta$. Zone 1 (c) and 3 (d) : $\bar{\Delta}=13 \Delta$ 


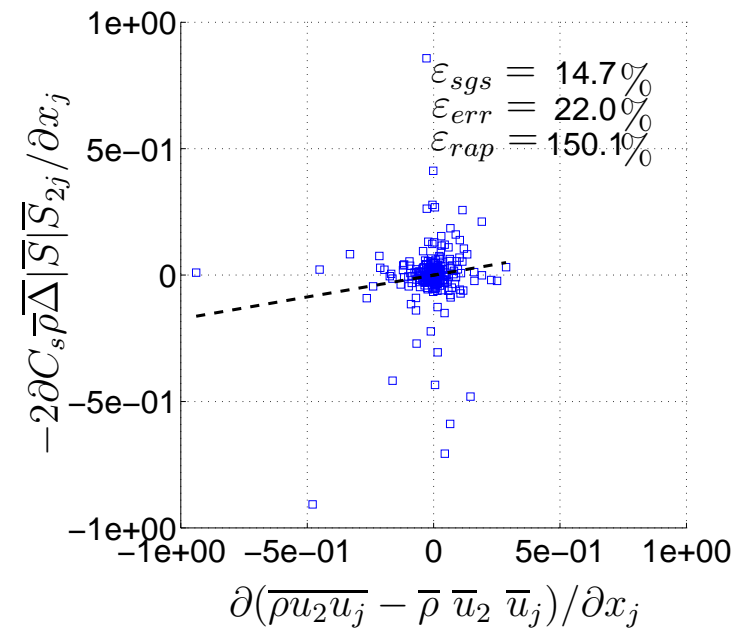

(a) Spanwise direction, zone 1.

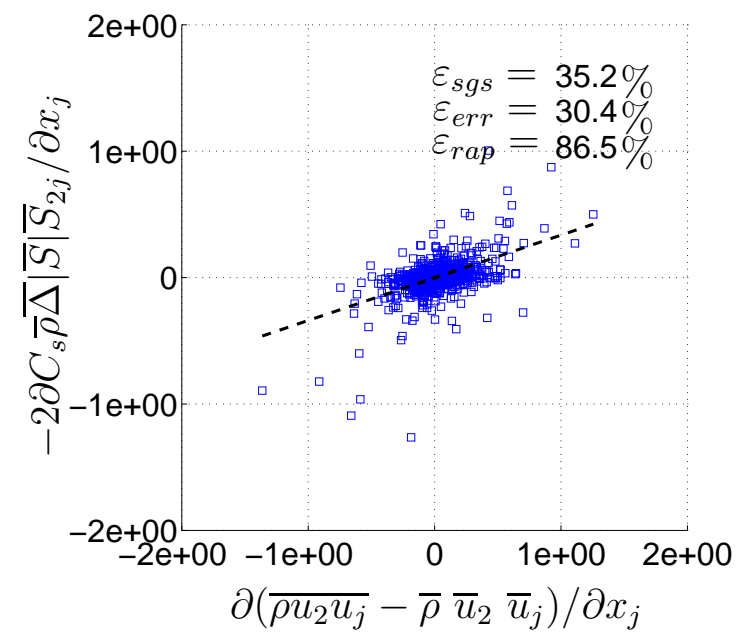

(c) Spanwise direction, zone 3 .

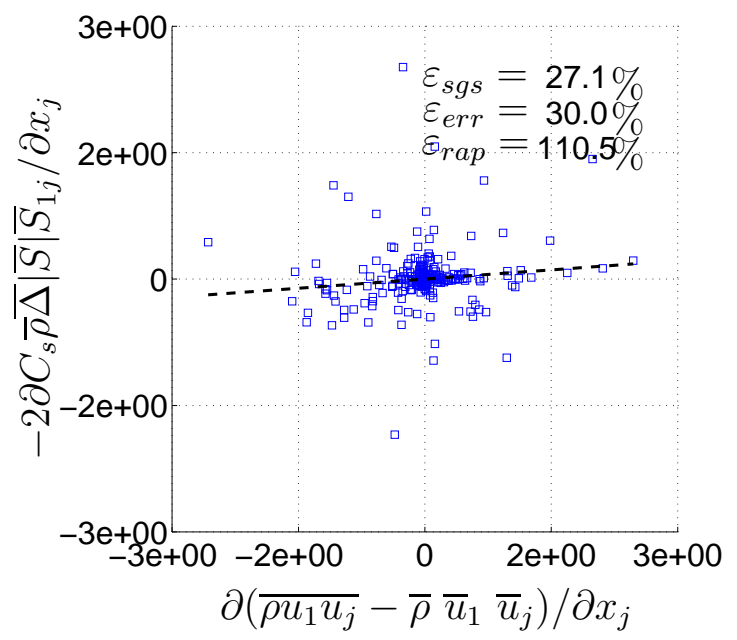

(b) Streamwise direction, zone 1.

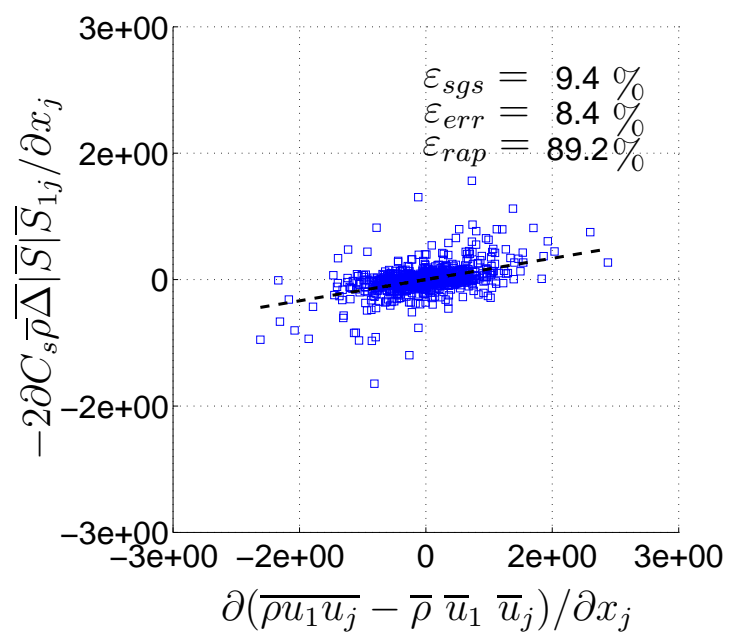

(d) Streamwise direction, zone 3.

Figure 11: Dispersion diagrams for the convective term with dynamic Smagorinsky procedure. $\bar{\Delta}=5 \Delta_{x}$. 


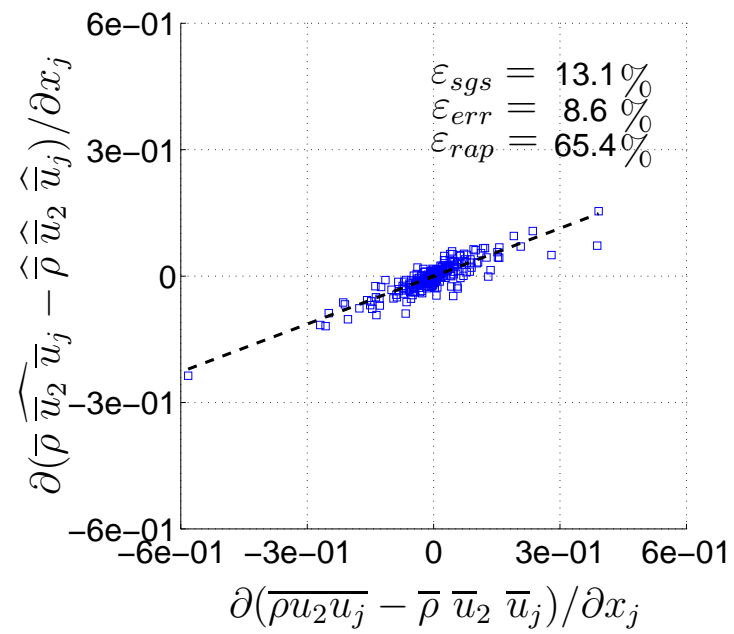

(a) Spanwise direction, zone 1.

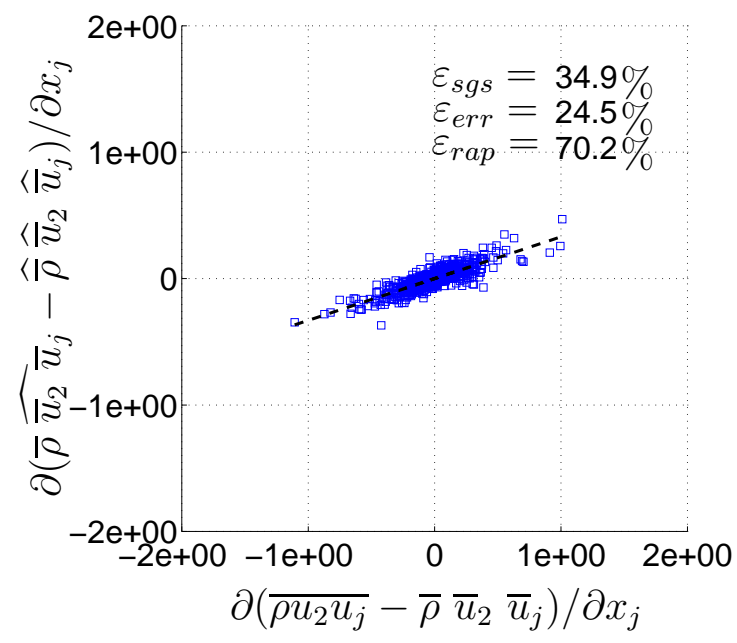

(c) Spanwise direction, zone 3 .

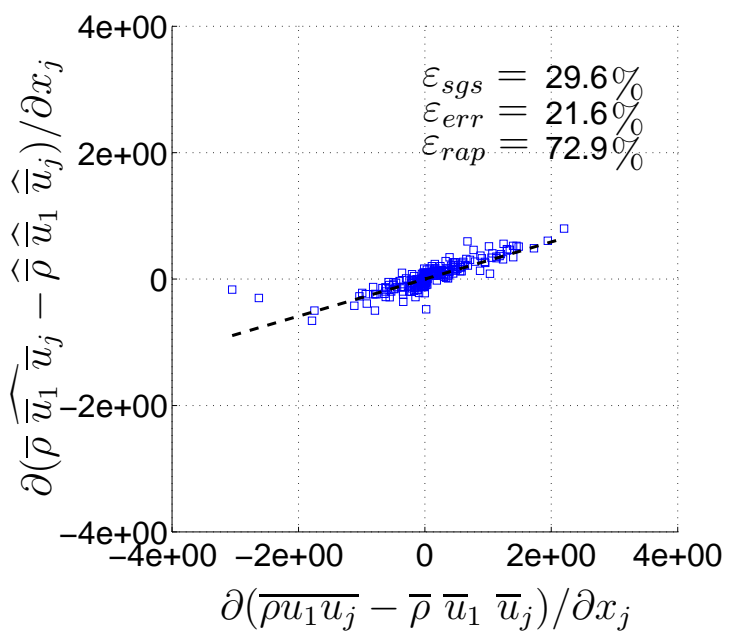

(b) Streamwise, zone 1.

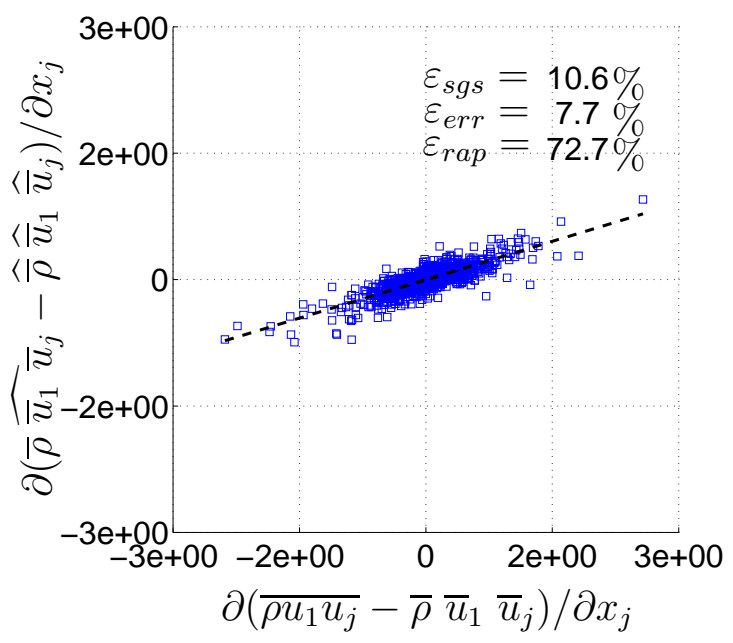

(d) Streamwise direction, zone 3.

Figure 12: Dispersion diagrams for the convective term with scale similarity assumption. $\bar{\Delta}=5 \Delta_{x}$. 


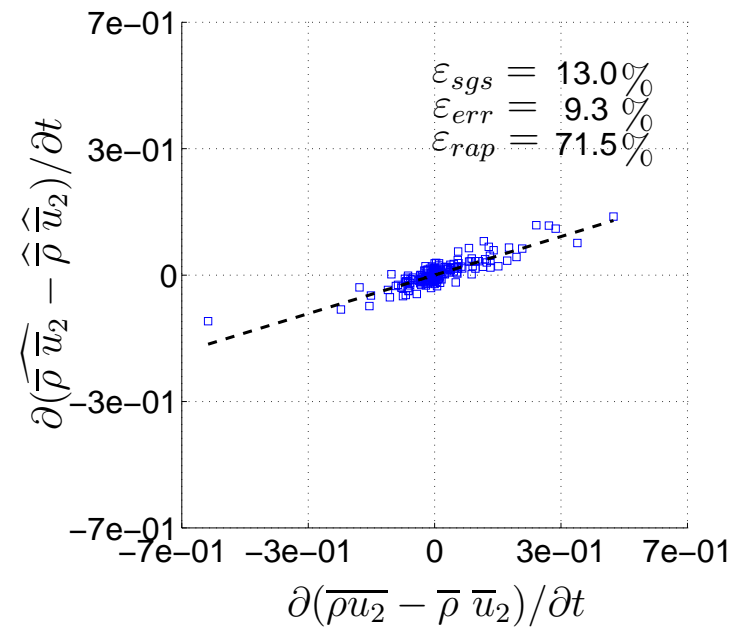

(a) Spanwise direction, zone 1.

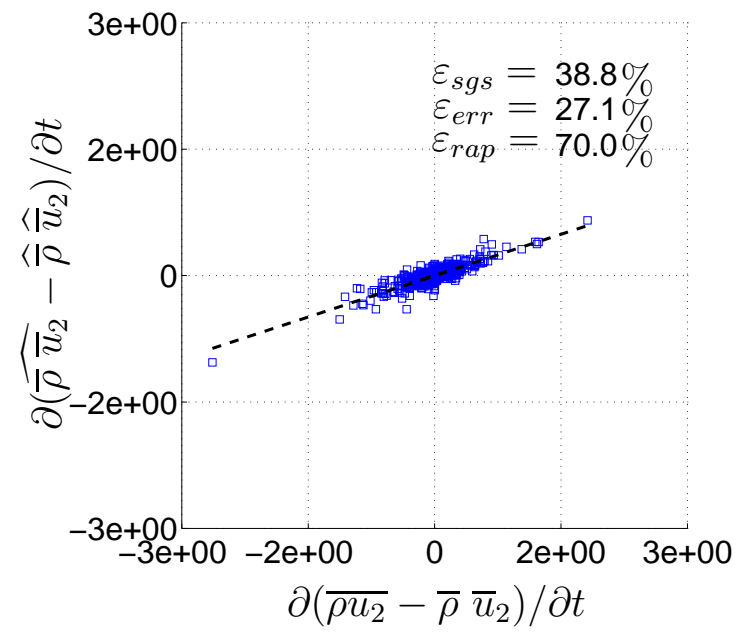

(c) Spanwise direction, zone 3 .

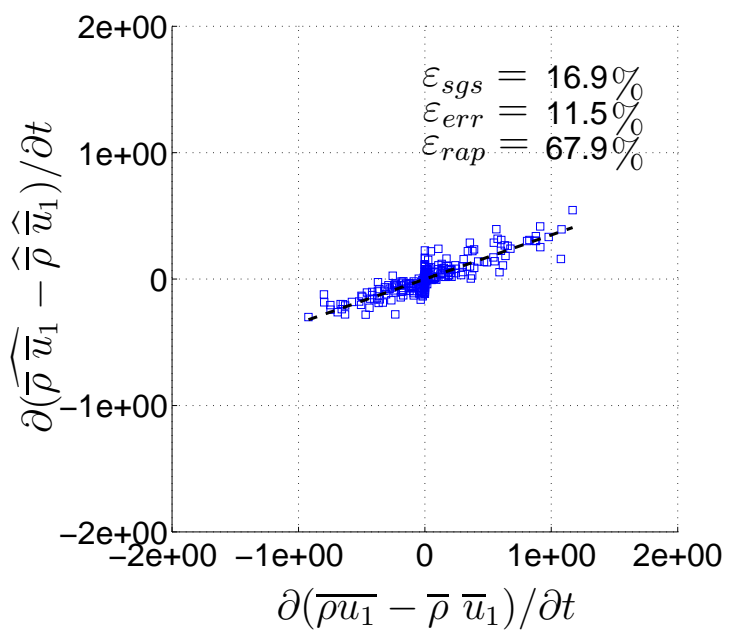

(b) Streamwise direction, zone 1.

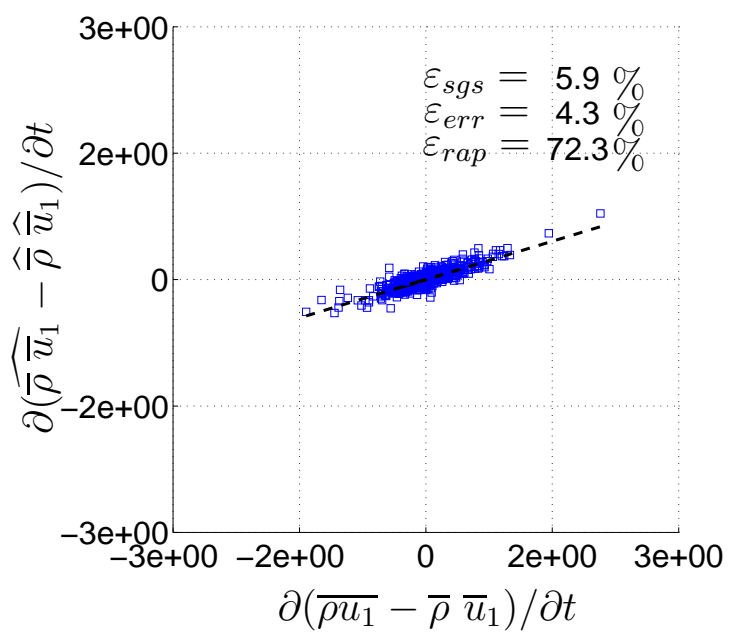

(d) Streamwise direction, zone 3.

Figure 13: Dispersion diagrams for the temporal term with scale similarity assumption. $\bar{\Delta}=5 \Delta_{x}$. 


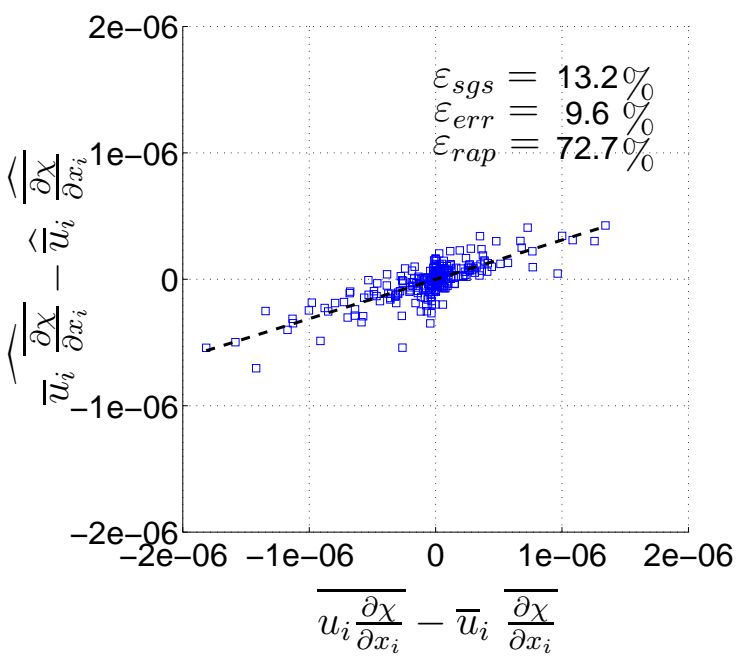

(a) $\bar{\Delta}=5 \Delta_{x}$, zone 1 .

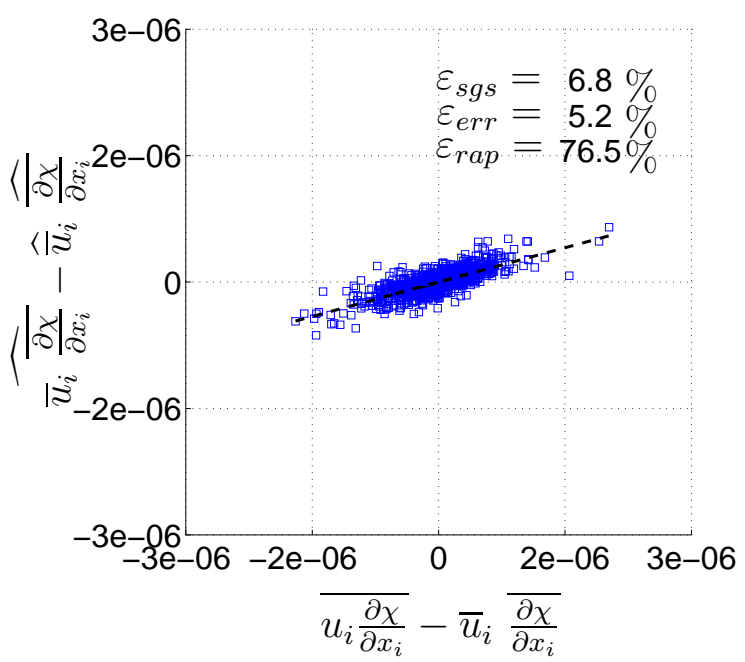

(c) $\bar{\Delta}=5 \Delta_{x}$, zone 3 .

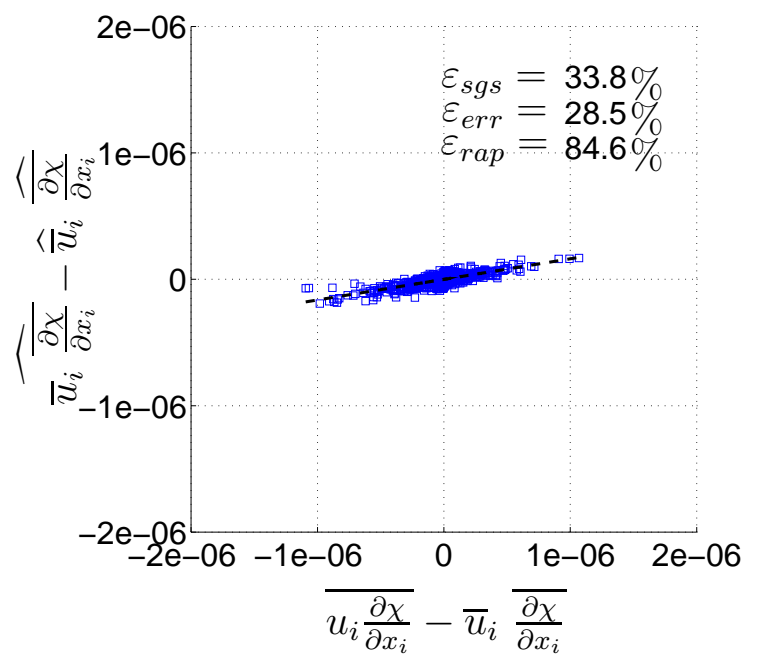

(b) $\bar{\Delta}=13 \Delta_{x}$, zone 1 .

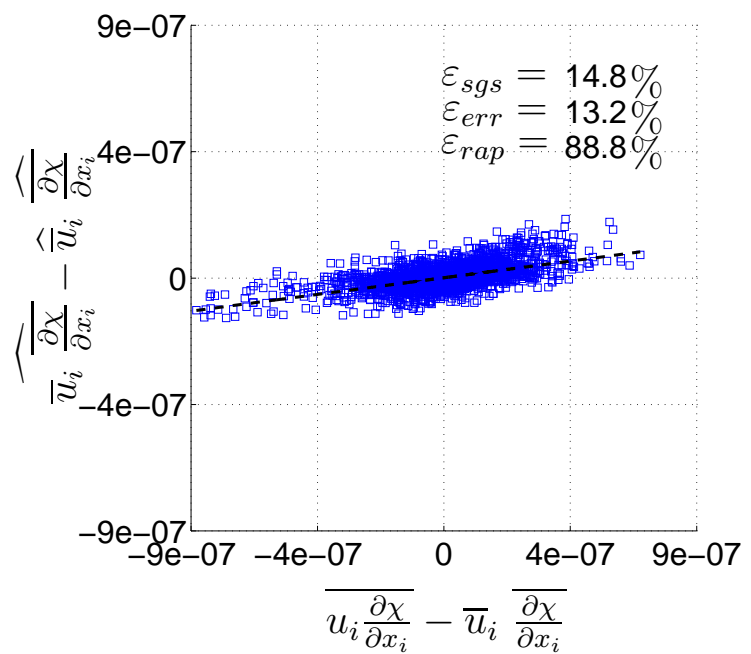

(d) $\bar{\Delta}=13 \Delta_{x}$, zone 3 .

Figure 14: Dispersion diagrams for the interfacial term with scale similarity assumption. 


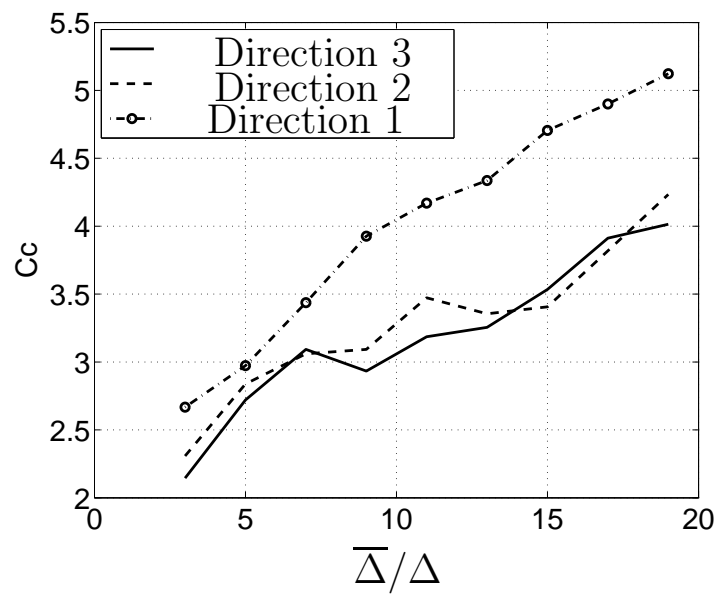

(a) Zone 1

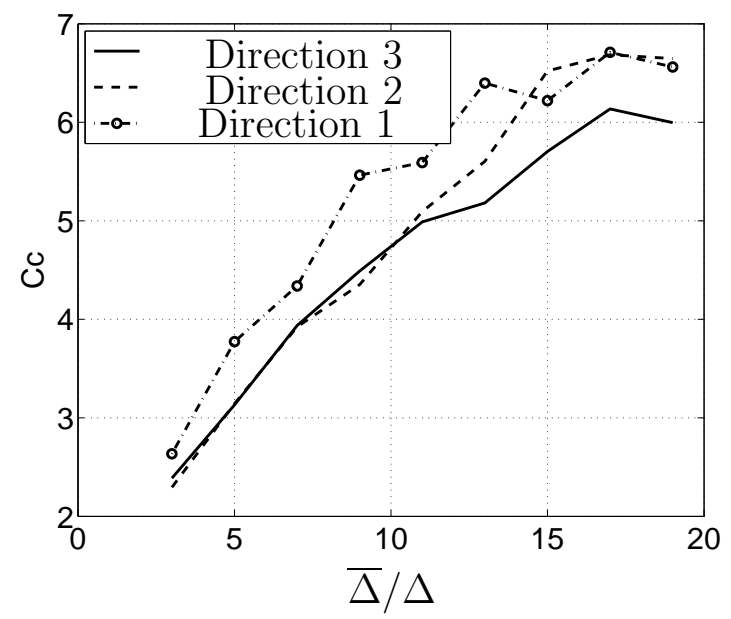

(b) Zone 3

Figure 15: Estimation of the scale similarity coefficients with respect to the subgrid filter size. 


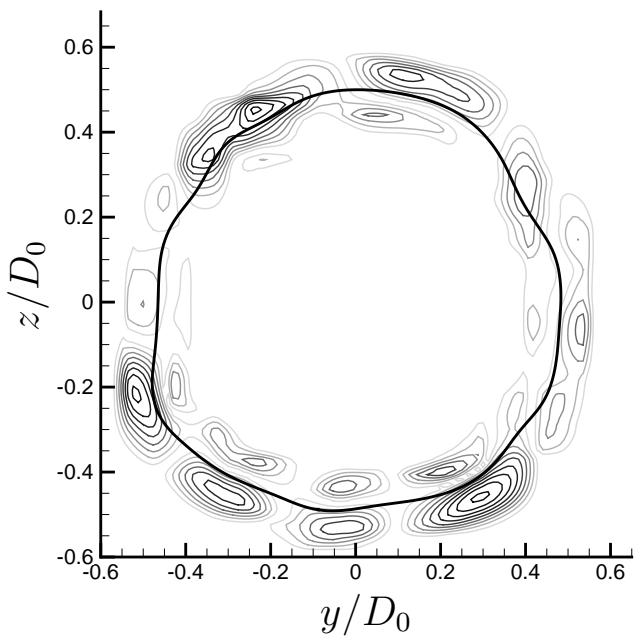

(a) Actual contribution $C_{1}$.

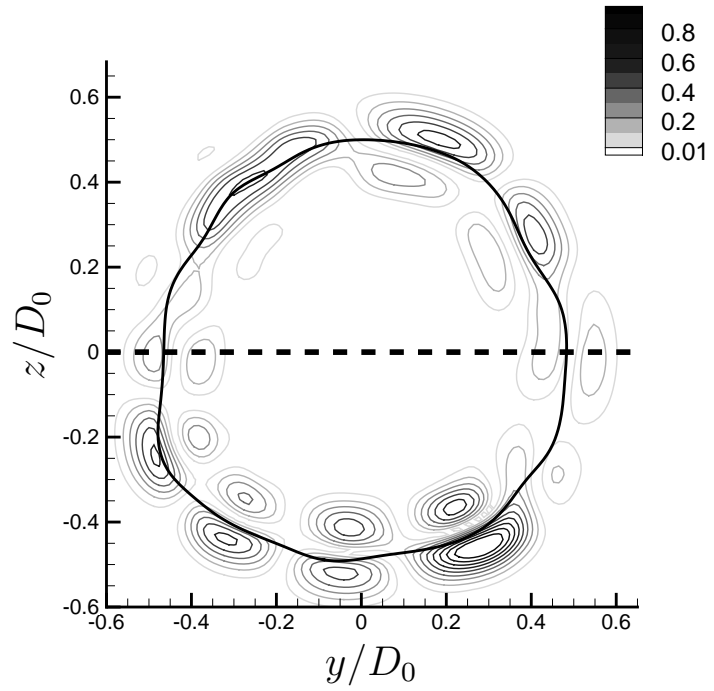

(b) Modeled contribution, $C_{1}^{m}$.

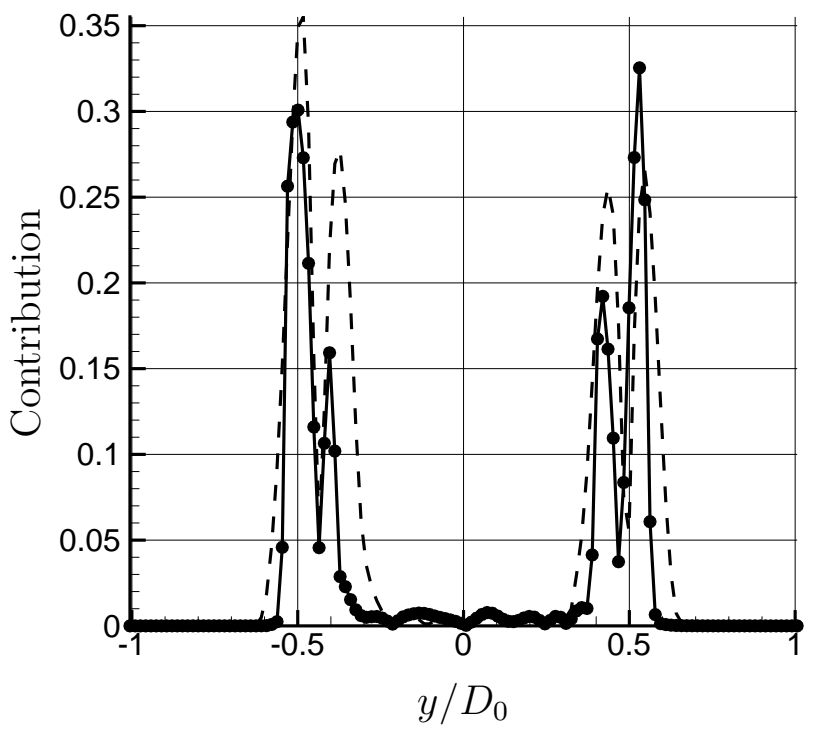

(c) profiles of $C_{1}$ (symbol line) and $C_{1}^{m}$ (dashed line) along $y$ direction.

Figure 16: $(\mathrm{a}-\mathrm{b}): y z$ cut in zone 1 of actua $\$$ and modeled convective subgrid contribution in the streamwise direction, respectively. (a): Actual term, $C_{1}$. (b) : modeled term $C_{1}^{m}$ with scale similarity assumption. (c) : profiles of exact and modeled contribution along $y$ direction (location visible in dashed line on (b)). $\bar{\Delta}=13 \Delta_{x}$. 


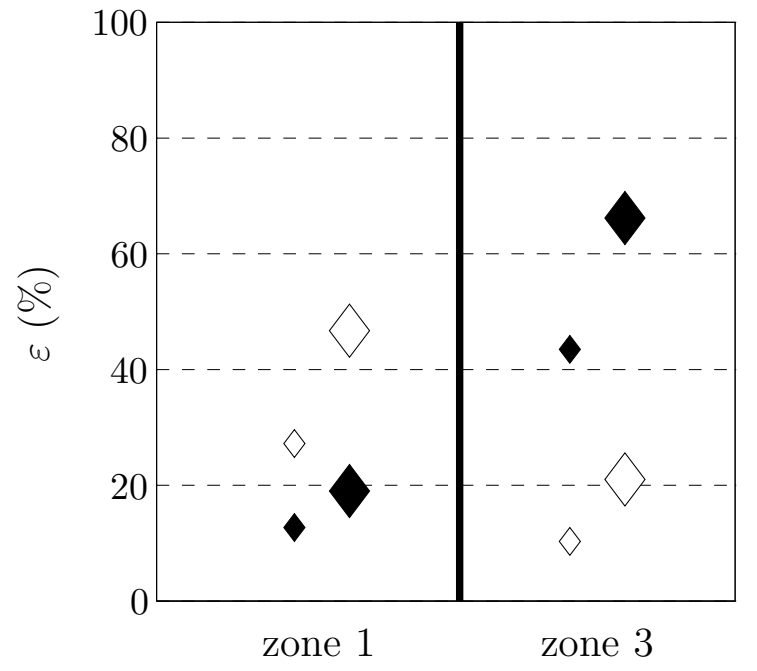

(a) $\varepsilon_{s g s}$

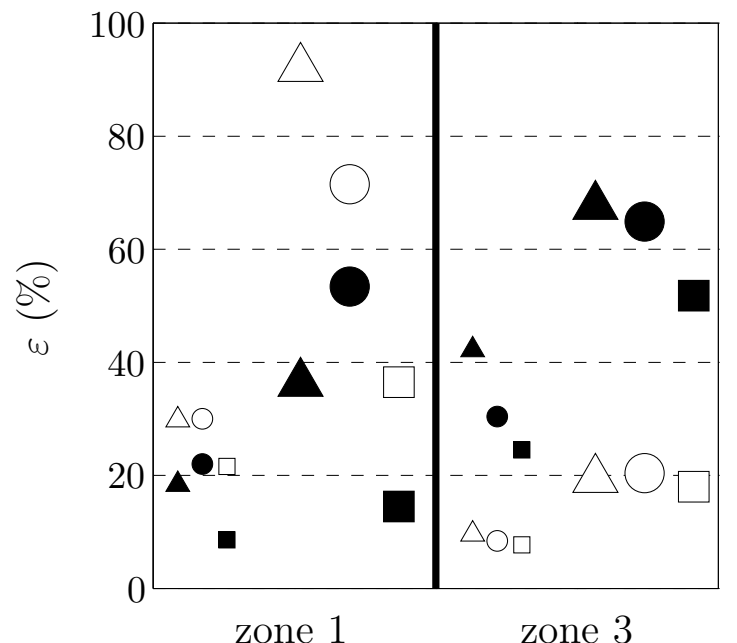

(b) $\varepsilon_{\text {err }}$

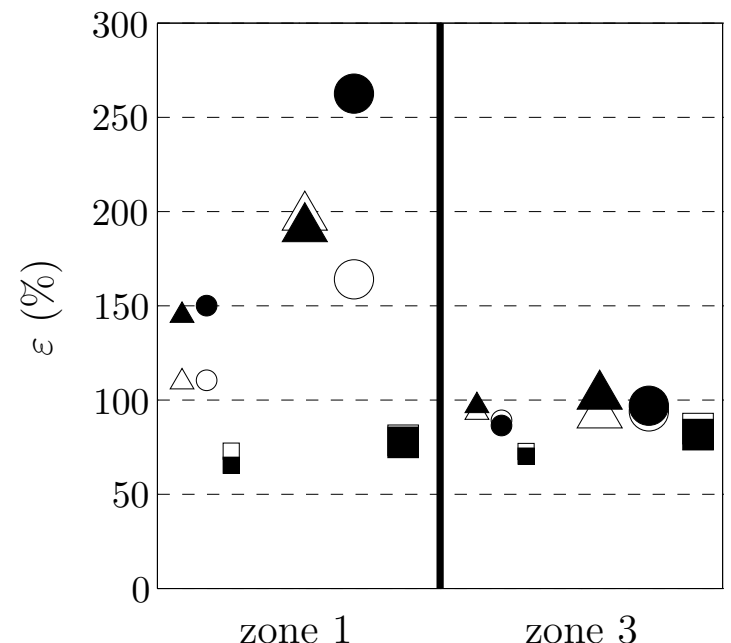

(c) $\varepsilon_{\text {rap }}$

Figure 17: Dispersion errors for the convective terms in zone 1 and 3. Empty and filled symbols are for the streamwise and spanwise contributions, respectively. The scale of the symbols is related to the filter size (i.e. smaller: SFS and biggest: LFS). $(\triangle)$ : Smagorinsky model; ( ○) : Eddy viscosity model, dynamic procedure. $(\square)$ : Scale similarity model. 


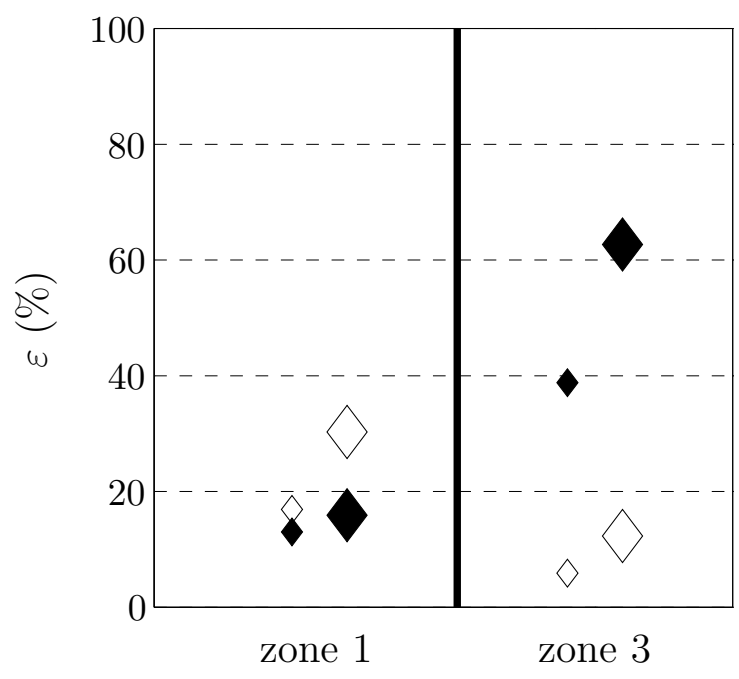

(a) $\varepsilon_{s g s}$

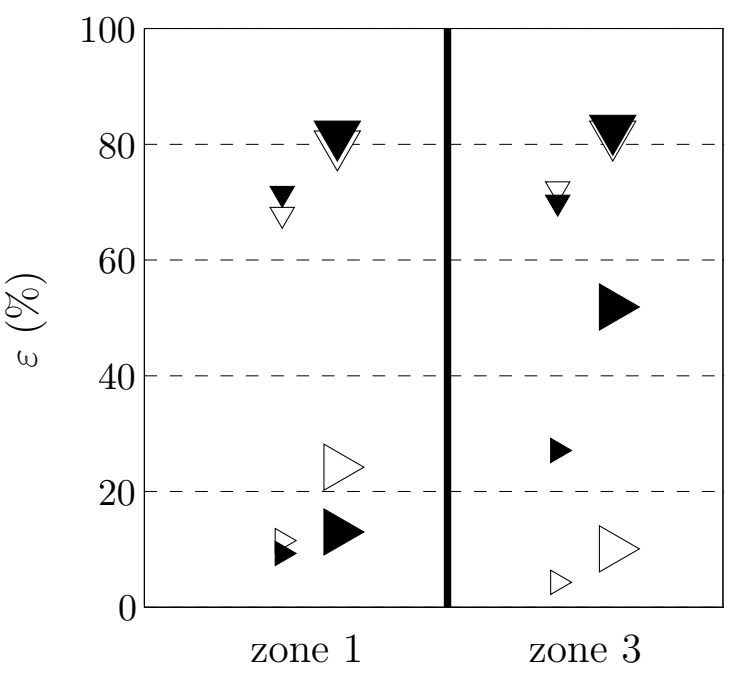

(b) $\varepsilon_{e r r}, \varepsilon_{r a p}$

Figure 18: Dispersion errors for the temporal term in zone 1 and 3 using the scale similarity assumption. Empty and filled symbols are for the streamwise and spanwise contributions, respectively. The scale of the symbols is related to the filter size (i.e. smaller: SFS and biggest: LFS). $(\triangleright): \varepsilon_{\text {err }} ;(\nabla): \varepsilon_{\text {rap }}$. 


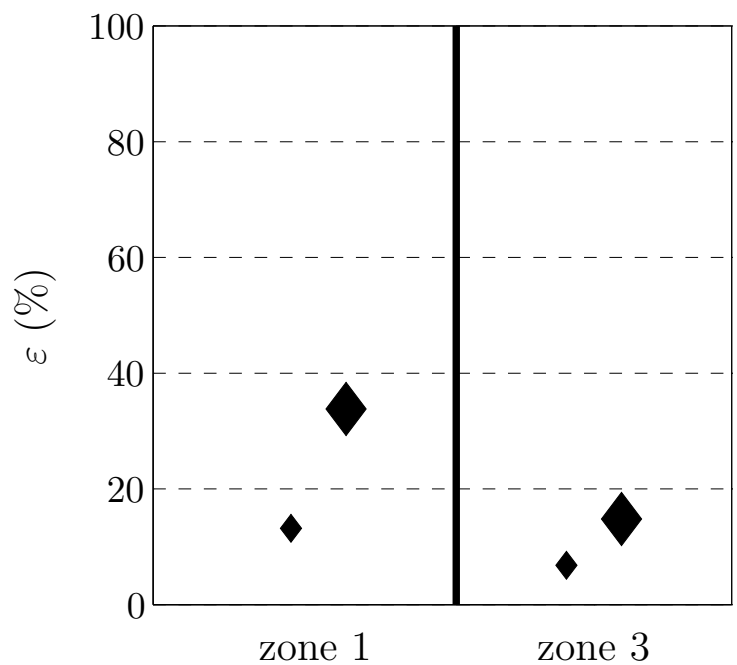

(a) $\varepsilon_{s g s}$

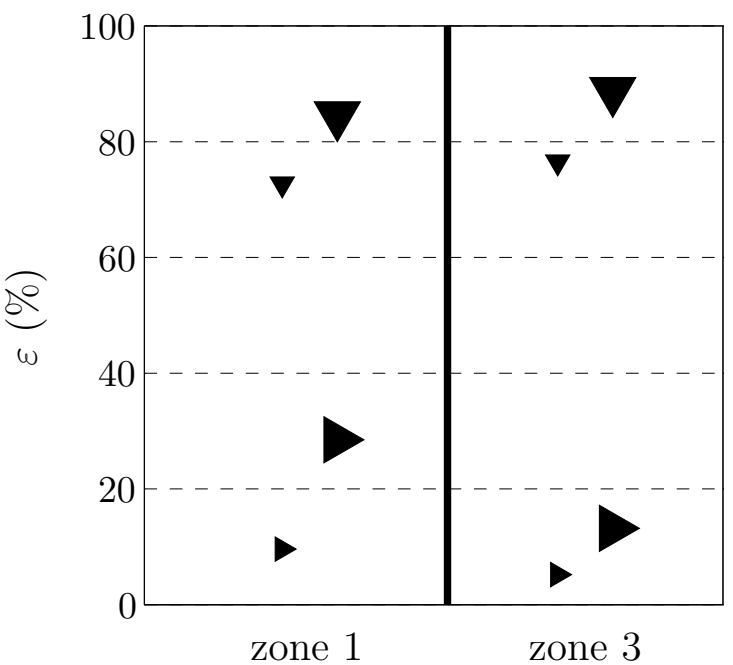

(b) $\varepsilon_{\text {err }}, \varepsilon_{\text {rap }}$

Figure 19: Dispersion errors for the interfacial term in zone 1 and 3 . The scale of the symbols is related to the filter size (i.e. smaller: SFS and biggest: LFS). ( $\boldsymbol{\nabla}): \varepsilon_{\text {err }} ;(\boldsymbol{\nabla})$ : $\varepsilon_{\text {rap }}$ 